\title{
Functional Application of G-Functions of Lorenzo and Hartley on the Free Convection Flow of Oldroyd-B Fluid with Ordinary and Fractional Techniques
}

\author{
Imran Siddique $\mathbb{B}^{1},{ }^{1}$ Sehrish Ayaz, ${ }^{1}$ Dalal Alrowaili $\mathbb{D}^{2},{ }^{2}$ and Sohaib Abdal ${ }^{3}$ \\ ${ }^{1}$ Department of Mathematics, University of Management and Technology, Lahore 54770, Pakistan \\ ${ }^{2}$ Mathematics Department, College of Science, Jouf University, P.O. Box: 2014, Sakaka, Saudi Arabia \\ ${ }^{3}$ School of Mathematics, Northwest University, No. 229 North Taibai Avenue, Xi'an 710069, Shaanxi Province, China \\ Correspondence should be addressed to Imran Siddique; imransmsrazi@gmail.com
}

Received 6 April 2021; Revised 5 June 2021; Accepted 28 August 2021; Published 8 September 2021

Academic Editor: Sheng Du

Copyright (c) 2021 Imran Siddique et al. This is an open access article distributed under the Creative Commons Attribution License, which permits unrestricted use, distribution, and reproduction in any medium, provided the original work is properly cited.

\begin{abstract}
In this article, free convection flow of an Oldroyd-B fluid (OBF) through a vertical rectangular channel in the presence of heat generation or absorption subject to generalized boundary conditions is studied. The fractionalized mathematical model is established by Caputo time-fractional derivative through mechanical laws (generalized shear stress constitutive equation and generalized Fourier's law). Closed form solutions for the velocity and temperature profiles are obtained via Laplace coupled with sine-Fourier transforms and have been embedded with regards to the special functions, namely, the generalized G-functions of Lorenzo and Hartley. Solutions of the known results from recently published work (Nehad et al. Chin. J. Phy., 65, (2020) 367-376) are recovered as limiting cases. Finally, the effects of fractional and various physical parameters are graphically underlined. Furthermore, a comparison between Oldroyd-B, Maxwell and viscous fluids (fractional and ordinary) is depicted. It is found that, for short time, ordinary fluids have greater velocity as compared to the fractional fluids.
\end{abstract}

\section{Introduction}

Fluids are classified in two categories with respect to the relation of shear stress and rate of deformation called Newtonian and non-Newtonian fluids. In non-Newtonian fluids, this relation is not linear, while in Newtonian fluids the relation of shear stress is linearly related to the rate of deformation. Several fluids have non-Newtonian behavior such as molten, salt solutions, custard, ketchup, blood, toothpaste, paint, and shampoo. A significant class of nonNewtonian fluids is viscoelastic fluids which show both adaptable and viscous property and numerous models of constitutive equations have been proposed to depict the transport conduct of these fluids. Oldroyd-B fluid (OBF) [1] is an exceptional non-Newtonian fluids and its transport conduct cannot be appropriately depicted by the usual relation between the shear rate and shear stress in a straightforward shear flow. Hence, numerous models of constitutive equations have been offered for these fluids in [2-8].

Free convection flows are of incredible viable significance to engineers and researchers on account of its allinclusive event in various mechanical applications, for example, cooling of electronic equipment's, solar amassers, fiber protection, and geothermal frameworks [9]. Because of its event in many engineering applications, numerous authors deliberate the free convection fluid flow models between two vertical parallel plates. The free convection fluid flow between two infinite vertical parallel plates is examined by Singh et al. [10]. Narahari [11] presented critical solution for the transient free convection flow between two vertical parallel plates within the sight of steady temperature and mass diffusion by using the Laplace transform method. Na et al. [12] found the closed form solutions of Maxwell free- 
convection fluid flow between vertical plates with damped shear and thermal flux. Zaib et al. [13] studied the natural convection flow of second-grade fluid with thermal radiation and damped thermal flux between vertical channels. Hajizadeh et al. [14] got the exact solutions for free convection flow of nanofluids between two vertical plates with damped thermal flux. Shao et al. [15] found the semianalytical solutions for hydromagnetic free-convection flow of viscous fluid between vertical parallel plates with damped thermal and mass fluxes. Seth et al. [16] have investigated the unsteady hydromagnetic flow formation with Hall Effect due to time-dependent free stream in a rotating medium. Seth et al. [17] have studied the effect of Hall current on MHD natural convection heat and mass transfer flow of rotating fluid past a vertical plate with ramped wall temperature. The natural convective heat transfer and nanofluid flows through a rectangular vertical channel under Robin-type conditions are concentrated by Ahmed et al. [18].

On the contrart, heat generation/absorption effects become relevant in many processes involving chemical reaction and dissociating fluids, since the flow and thermal fields can be greatly influenced by the heat source/sink term. Jha et al. [19] examined the effect of heat generation/absorption on flow formation in a parallel plate channel with ramped temperatures. In a related work, Jha and Aina [20] obtained the exact solution for heat generating/absorbing fluid in a cylinder under time periodic boundary conditions in the presence of magnetic field. They found out that the rate of heat transfer is significantly declined with increase in heat sink, while the contrast is true in presence of heat source. Jha et al. [21] presented the exact solution responsible for the mixed convection in an annular geometry when the fluid is either heat generating or heat absorbing. In another work, Saba et al. [22] presented a study on thermal analysis of nanofluid in the presence of internal heat generation/absorption. Other related discussion can be found in [23-28].

Fractional calculus $[29,30]$ has been utilized effectively in the depiction of viscoelasticity. Mostly, these constitutive equations are obtained from known models via substituting time ordinary derivatives of stress and strain by derivatives of fractional order. The generality permits one to express indeed noninteger-order integral or derivatives. Recently, numerous specialists have considered various issues associated to such fluids. Hyder Ali Muttaqi Shah [31] examined the flows of generalized OBF between two side walls perpendicular to the plate. Akgül et al. [32, 33] analyzed the MHD Couette flows by fractal-fractional differential operators. Some accelerated flows of a generalized OBF are discussed by Fetecau et al. [34]. Siddique and Akgül [35] examined the blood liquor model via nonlocal and singular constant proportional Caputo hybrid differential operator. Khan et al. [36] examined the MHD flow of a generalized $\mathrm{OBF}$ in a round funnel. Siddique and Sajid [37] founnd the exact solutions for the unsteady axial flow of non-Newtonian fluids through a circular cylinder. MHD OBF through fractional calculus approach under several conditions has been examined by Liu et al. [38]. Solutions for OBF between two oscillating plates with transverse magnetic field have been investigated by Bose and Basu [39]. Zhao et al. [40] carried out numerical solutions for fractional OBF in porous medium under the influence of heat transfer. Zafar et al. [41] contemplated an OBF for circular cylinders with nonintegerorder derivatives. Zhang et al. [42] examined the analytical solutions for time-fractional OBF utilizing the definition of Caputo derivative. As of late, Riaz and Saeed [43] evaluated the conduct of MHD OBF under slip condition with the assistance of integer order, Caputo-Fabrizio, and Atangana-Baleanu fractional derivatives. Wang et al. [44] acquired the semianalytical solutions for velocity field and tangential stress correspond to fractional Oldroyd-B fluid in an annulus by Laplace transforms and modified Bessel equation. Kamran et al. [45] find the exact solutions for the unsteady rotational flow of an Oldroyd-B fluid with fractional derivative through an infinite circular cylinder by means of the finite Hankel and Laplace transforms. In all these studies the ordinary derivatives are falsely supplanted by fractional derivatives. Some recent works were done by specialists, and they introduced fractional derivatives through mechanical laws to governing equations [12-15, 46-48].

In the current paper, as uniqueness, we build up a nonlocal mathematical model in which the thermal transport is depicted by the fractional Fourier's law. The new fractional constitutive equations are characterized with the time-fractional Caputo derivative; hence, they give a powerlaw damping to the temperature and velocity. These realities were the significant reason of the lack of literature on the study of OBFs between two-side walls under general boundary conditions. In this work, we acquire the closed form solutions of the generalized convection flows of OBF between two parallel plates under general boundary conditions by utilizing the Laplace transform coupled with the finite sine-Fourier transform. The solutions are uttered in the form of generalized G-functions of Lorenzo and Hartley [49]. The impacts of fractional and physical parameters are graphically outlined. In addition, the limiting cases of the current outcomes compare to the solutions for fractional Maxwell, ordinary Maxwell, Oldroyd-B, and Newtonian fluids.

\section{Mathematical Modeling}

Let us consider the unsteady free-convection Oldroyd-B, incompressible, and electrically conducting fluid flow between two infinite nonconducting parallel vertical plates separated by a distance $d$. The flow geometry in fixed Cartesian coordinate system $x, \zeta_{1}^{\mp}$ and $z$ is shown in Figure 1. The $x$-axis is taken along the plates in the direction of fluid flow with the velocity $u^{+}$and the $\zeta_{1}^{\mp}$-axis is taken perpendicular to the plates.

Since the channel is infinitely extended in the $x$ and $z$ directions, we can assume that all physical entities describing the fluid motion and heat transfer are the functions of $\zeta_{1}^{\mp}$ and $t_{1}^{\mp}$ only. At first, both the plates and the enclosed fluid are at rest at the ambient temperature $T_{a}$. After this moment, the left and right plates starts to slid in their own planes along $x$-direction with the velocities $U_{0} g_{1}^{\mp}\left(t_{1}^{\mp}\right)$ and $U_{0} g_{2}^{\mp}\left(t_{1}^{\mp}\right)$; individually, plates are maintained at temperatures $T_{a}+\left(T_{b}-T_{a}\right) f_{1}^{\mp}\left(t_{1}^{\mp}\right) \quad$ and $\quad T_{a}+\left(T_{b}-T_{a}\right) f_{2}^{\mp}\left(t_{1}^{\mp}\right)$, 


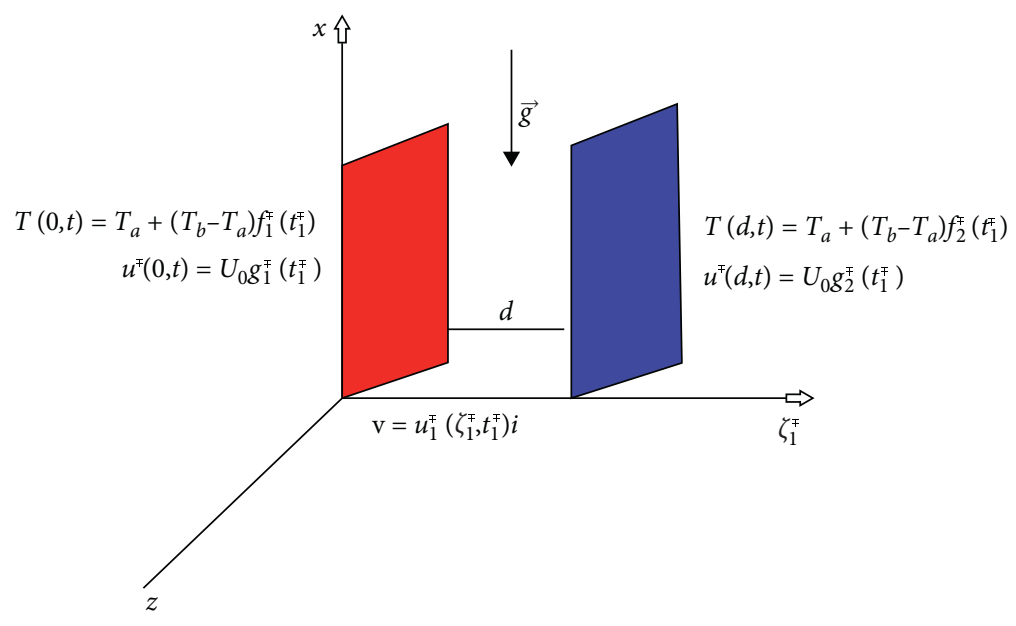

Figure 1: Flow geometry.

respectively, where $U_{0}$ is a constant with dimension of velocity; the functions $f_{i}(\cdot)$ and $g_{i}(\cdot), i=1,2$, are piecewise continuous and exponential order functions at infinity with $f_{i}(0)=g_{i}(0)=0, i=1,2$.

We made the following assumptions for the fluid flows:

(i) Velocity is only a function of material coordinate $\zeta_{1}^{\mp}$ and special coordinate $t_{1}^{\mp}$, i.e., $\mathbf{v}=\left(u_{1}^{\mp}\left(\zeta_{1}^{\mp}, t_{1}^{\mp}\right), 0,0\right)$

(ii) Joule heating and viscous dissipation in energy equations are negligible

In the absence of a pressure gradient in the flow direction and utilizing the typical Boussinesq's approximation, the governing equations for the unsteady free-convection flow of OBF through the vertical channel communicated by the subsequent equations $[50,51]$ are the linear momentum equation,

$$
\rho \frac{\partial u_{1}^{\mp}\left(\zeta_{1}^{\mp}, t_{1}^{\mp}\right)}{\partial t_{1}^{\mp}}=\frac{\partial \tau_{1}^{\mp}\left(\zeta_{1}^{\mp}, t_{1}^{\mp}\right)}{\partial \zeta_{1}^{\mp}}+g \rho \beta_{T}\left[T^{\mp}\left(\zeta_{1}^{\mp}, t_{1}^{\mp}\right)-T_{a}\right],
$$

the constitutive equation,

$$
\left(1+\lambda^{ \pm} \frac{\partial}{\partial t_{1}^{\mp}}\right) \tau_{1}^{\mp}\left(\zeta_{1}^{\mp}, t_{1}^{\mp}\right)=\mu\left(1+\lambda_{r}^{\mp} \frac{\partial}{\partial t_{1}^{\mp}}\right) \frac{\partial u_{1}^{\mp}\left(\zeta_{1}^{\mp}, t_{1}^{\mp}\right)}{\partial \zeta_{1}^{\mp}},
$$

the thermal balance equation,

$$
\rho c_{p} \frac{\partial T^{\mp}\left(\zeta_{1}^{\mp}, t_{1}^{\mp}\right)}{\partial t_{1}^{\mp}}=-\frac{\partial q_{1}\left(\zeta_{1}^{\mp}, t_{1}^{\mp}\right)}{\partial \zeta_{1}^{\mp}}-Q_{0}\left[T^{ \pm}\left(\zeta_{1}^{\mp}, t_{1}^{\mp}\right)-T_{a}\right],
$$

and Fourier's law,

$$
q_{1}\left(\zeta_{1}^{\mp}, t_{1}^{\mp}\right)=-k \frac{\partial T^{\mp}\left(\zeta_{1}^{\mp}, t_{1}^{\mp}\right)}{\partial \zeta_{1}^{\mp}},
$$

under corresponding initial and boundary conditions:

$$
\begin{aligned}
& T^{\mp}\left(\zeta_{1}^{\mp}, 0\right)=T_{a}, \\
& u_{1}^{\mp}\left(\zeta_{1}^{\mp}, 0\right)=0, \quad 0 \leq \zeta_{1}^{\mp} \leq d, \\
& T^{\mp}\left(0, t_{1}^{\mp}\right)=T_{a}+\left(T_{b}-T_{a}\right) f_{1}^{\mp}\left(t_{1}^{\mp}\right), \\
& T^{\mp}\left(d, t_{1}^{\mp}\right)=T_{a}+\left(T_{b}-T_{a}\right) f_{2}^{\mp}\left(t_{1}^{\mp}\right), \\
& u_{1}^{\mp}\left(0, t_{1}^{\mp}\right)=U_{0} g_{1}^{\mp}\left(t_{1}^{\mp}\right), \\
& u_{1}^{\mp}\left(d, t_{1}^{\mp}\right)=U_{0} g_{2}^{\mp}\left(t_{1}^{\mp}\right), \quad t_{1}^{\mp} \geq 0 .
\end{aligned}
$$$$
T^{\mp}\left(d, t_{1}^{\mp}\right)=T_{a}+\left(T_{b}-T_{a}\right) f_{2}^{\mp}\left(t_{1}^{\mp}\right), \quad t_{1}^{\mp} \geq 0,
$$

Introducing the following nondimensionless variables, functions, and parameters, 


$$
\begin{aligned}
& y=\frac{\zeta_{1}^{\mp}}{d} \\
& t=\frac{v t_{1}^{\mp}}{d^{2}} \\
& u=\frac{u_{1}^{\mp}}{U_{0}}, \\
& T=\frac{T^{\mp}-T_{a}}{T_{b}-T_{a}}, \\
& q=\frac{q_{1} d}{k\left(T_{b}-T_{a}\right)}, \\
& \tau=\frac{\tau_{1}^{\mp} d}{\mu U_{0}}, \\
& \lambda=\frac{\nu \lambda^{\mp}}{d^{2}}, \\
& \lambda_{r}=\frac{v \lambda_{r}^{\mp}}{d^{2}}, \\
& Q_{0}=\frac{Q d^{2}}{k}, \\
& \operatorname{Pr}=\frac{\mu c_{p}}{k}, \\
& \mathrm{Gr}=\frac{g \beta_{T}\left(T_{b}-T_{a}\right) d^{2}}{v U_{0}}, \\
& f_{j}^{\mp}\left(t_{1}^{\mp}\right)=f_{j}\left(\frac{\mathrm{d}^{2} t}{v}\right), \\
& g_{j}^{\mp}\left(t_{1}^{\mp}\right)=g_{j}\left(\frac{\mathrm{d}^{2} t}{v}\right), \quad j=1,2,
\end{aligned}
$$

$$
q(y, t)=-\frac{\partial T(y, t)}{\partial y} .
$$

The partial differential equations (9)-(12) are taken along with the following dimensionless initial and boundary conditions:

$$
\begin{aligned}
& u(y, 0)=0, \\
& T(y, 0)=0, \quad 0 \leq y \leq 1, \\
& T(0, t)=f_{1}(t), \\
& T(1, t)=f_{2}(t), \quad t \geq 0, \\
& u(0, t)=g_{1}(t), \\
& u(1, t)=g_{2}(t), \quad t \geq 0 .
\end{aligned}
$$

In the following, we develop a fractional model in which the classical constitutive equations (10) and (12) are generalized by using the constitutive shear stress equation:

$$
\left(1+\lambda \frac{\partial}{\partial t}\right) \tau(y, t)=\left(1+\lambda_{r} \frac{\partial}{\partial t}\right){ }^{C} D_{t}^{1-\alpha}\left(\frac{\partial u(y, t)}{\partial y}\right), \quad 0<\alpha \leq 1,
$$

proposed by Scott-Blair [52], respectively, and the generalized Fourier's law,

$$
q(y, t)={ }^{C} D_{t}^{1-\beta}\left(\frac{\partial T(y, t)}{\partial y}\right), \quad 0<\beta \leq 1,
$$

proposed by Povstenko [53] and Hristov [54], respectively. In the above constitutive equations, ${ }^{C} D_{t}^{\alpha}(\cdot)$ denotes the time-fractional Caputo derivative defined by $[55,56]$. Hence,

$$
\begin{aligned}
{ }^{C} D_{t}^{\alpha} u(y, t) & =\frac{1}{\Gamma(1-\alpha)} \int_{0}^{t} \dot{u}(y, s)(t-s)^{-\alpha} \mathrm{d} s \\
& =\left(h_{\alpha}(t) * \dot{u}(y, t)\right), \quad 0 \leq \alpha \leq 1,
\end{aligned}
$$

where $h_{\alpha}(t)=\left(t^{-\alpha} / \Gamma(1-\alpha)\right)$ is the singular power-law kernel for $\dot{u}(y, s)=\left.(\partial u(y, t) / \partial t)\right|_{t=s}$.

Furthermore, using the second form of the time-fractional Caputo derivative from equation (18) and one of the following properties of $h_{\alpha}(\cdot)$ is defined as

$$
\begin{aligned}
L\left\{h_{\alpha}(t)\right\} & =\frac{1}{s^{1-\alpha}}, \\
\left(h_{1-\alpha} * h_{\alpha}\right)(t) & =1, \\
h_{0}(t) & =L^{-1}\left\{\frac{1}{s}\right\}=1, \\
h_{1}(t) & =L^{-1}\{1\}=\delta(t),
\end{aligned}
$$

where $L\{\cdot\}$ denotes the Laplace transform, $\delta(\cdot)$ is Dirac's distribution, and $s$ is the transform parameter, and it is easy to show that

$$
\frac{\partial T(y, t)}{\partial t}=-\frac{1}{\operatorname{Pr}} \frac{\partial q(y, t)}{\partial y}-Q T(y, t),
$$




$$
\begin{aligned}
& { }^{C} D_{t}^{0} f(y, t)=f(y, t)-f(y, 0), \\
& \left({ }^{C} D_{t}^{0} f(y, t)=f(y, t), \text { if } f(y, 0)=0\right), \\
& { }^{C} D_{t}^{1} f(y, t)=\frac{\partial f(y, t)}{\partial t} .
\end{aligned}
$$

Using properties (20), for $\alpha=\beta=1$, equations (16) and (17) reduce to the classical forms (10) and (12).

It is important to specify that the models based on fractional constitutive equations are more general than those described by constitutive equations with integer derivatives.

For this, let us analyze the constitutive equations for the thermal flux (12) and (17). Equation (12) corresponding to Fourier's law is a local form in time and space. Indeed, the value of the heat flux in a position $y$ at time $t$ is determined by the value of the temperature gradient in the same spatial position and at the same time.

The generalized constitutive equation (17), written in the equivalent form $q(y, t)=-(1 / \Gamma(\beta))(\partial / \partial y) \int_{0}^{t}(t-s)^{\beta-1}$ $\partial T(y, t) /\left.\partial t\right|_{t=s} \mathrm{~d} s$, shows that the value of the thermal flux in position $y$ at time $t$ is influenced by the temperature history; therefore, the values of the time derivative of temperature for all past and present times determine the thermal flux at the moment $t$. Such types of constitutive equations describe the thermal processes with time nonlocality. The function $h_{1-\beta}(t)=\left(t^{\beta-1} / \Gamma(\beta)\right)$ is the time-nonlocality kernel (the weight function).

The processes with time nonlocality are called processes with memory. The classical Fourier's law describes processes with "instantaneous memory" whose time nonlocality kernel being Dirac's distribution. If the weight function is constant, the process is called the "full memory" process, i.e., there is no fading of memory.

A similar discussion can be made regarding the generalized constitutive equation (16). The difference, in this case, is about the memory of the material deformation. It can be seen from equation (16) that the history of the strain rate influences the stress at the moment $t$.

Eliminating $\tau$ from equations (9) and (16) and $q$ from equations (11) and (17), we obtain

$$
\begin{gathered}
\left(1+\lambda \frac{\partial}{\partial t}\right) \frac{\partial u(y, t)}{\partial t}=\left(1+\lambda_{r} \frac{\partial}{\partial t}\right){ }^{C} D_{t}^{1-\alpha}\left(\frac{\partial^{2} u(y, t)}{\partial y^{2}}\right)+\operatorname{Gr}\left(1+\lambda \frac{\partial}{\partial t}\right) T(y, t), \\
\frac{\partial T(y, t)}{\partial t}=\frac{1}{\operatorname{Pr}} D_{t}^{1-\beta}\left(\frac{\partial^{2} T(y, t)}{\partial y^{2}}\right)-Q T(y, t) .
\end{gathered}
$$

In order to obtain the equivalent forms of equations (21) and (22), we recall the time-fractional integral operator:

$$
J_{t}^{\alpha} f(y, t)=\left(h_{1-\alpha} * f\right)(t)=\frac{1}{\Gamma(\alpha)} \int_{0}^{t} f(y, s)(t-s)^{\alpha-1} \mathrm{~d} s,
$$

which is the left-inverse operator of the derivative operator ${ }^{C} D_{t}^{\alpha}(\cdot)$. Indeed, using equations (18), (19), and (23), we obtain that

$$
\begin{aligned}
\left(J_{t}^{\alpha}{ }^{C}{ }^{C} D_{t}^{\alpha}\right) f(y, t) & =J_{t}^{\alpha}\left({ }^{C} D_{t}^{\alpha} f(y, t)\right)=\left[h_{1-\alpha} *\left(h_{\alpha} * \dot{f}\right)\right](t) \\
& =\left[\left(h_{1-\alpha} * h_{\alpha}\right) * \dot{f}\right](t)=[1 * \dot{f}](t)=f(y, t)-f(y, 0), \\
& J_{t}^{1-\alpha} \dot{f}(y, t)=\left(h_{\alpha} * \dot{f}\right)(t)={ }^{C} D_{t}^{\alpha} f(y, t) .
\end{aligned}
$$

which implies

$$
\left(J_{t}^{\alpha}{ }^{\circ}{ }^{C} D_{t}^{\alpha}\right) f(y, t)=f(y, t), \quad \text { if } f(y, 0)=0 .
$$

Moreover, using equations (18) and (23), it results that
Now, applying the fractional integral operators $J_{t}^{1-\alpha}$ and $J_{t}^{1-\beta}$ to equations (21) and (22) and using properties (24)-(26) together with the initial condition, we obtain the following fractional differential equations: 


$$
\begin{aligned}
\left(1+\lambda \frac{\partial}{\partial t}\right){ }^{C} D_{t}^{\alpha} u(y, t) & =\left(1+\lambda_{r} \frac{\partial}{\partial t}\right) \frac{\partial^{2} u(y, t)}{\partial y^{2}}+\operatorname{GrJ}_{t}^{1-\alpha}\left(1+\lambda \frac{\partial}{\partial t}\right) T(y, t), \\
{ }^{C} D_{t}^{\beta} T(y, t) & =\frac{1}{\operatorname{Pr}} \frac{\partial^{2} T(y, t)}{\partial y^{2}}-Q J_{t}^{1-\beta} T(y, t) .
\end{aligned}
$$

To determine the solutions of equations (27) and (28), we will employ the Laplace and finite Fourier transform.

The mathematical models considered in this paper describe real problems of fluid flow and heat transfer in a rectangular channel. Therefore, we consider for the study problems in which the temperature and velocity fields are functions that are at least twice differentiable in relation to the variables on which they depend. It is also considered that the functions that describe the movement and heat transfer have additional properties so that the existence of the Laplace and Fourier transforms to be provided.

Therefore, for the studied problems, we search solutions in the class of functions that satisfy the above properties.

\section{Solution of the Problem}

3.1. Temperature Distribution. Applying the Laplace transform to equations (28) and (14), using initial condition (13), we obtain

$$
\begin{aligned}
s^{\beta} \bar{T}(y, s) & =\frac{1}{\operatorname{Pr}} \frac{\partial^{2} \bar{T}(y, s)}{\partial y^{2}}-Q s^{\beta-1} \bar{T}(y, s), \\
\bar{T}(0, s) & =F_{1}(s), \\
\bar{T}(1, s) & =F_{2}(s),
\end{aligned}
$$

where $\bar{T}(y, s)=\int_{0}^{\infty} \bar{T}(y, t) e^{-s t} \mathrm{~d} t, s$ is Laplace transform parameter, and $L\left\{f_{i}(t)\right\}=F_{i}(s) ; i=1,2$.

Applying the finite sine-Fourier transform to differential equation (29) subject to the conditions in equation (30), we obtain

$$
\widetilde{T}_{s}(n, s)=\frac{(n \pi)\left(F_{1}(s)+(-1)^{n+1} F_{2}(s)\right)}{\operatorname{Pr} s^{\beta}+Q s^{\beta-1}+(n \pi)},
$$

where $\widetilde{\bar{T}}_{s}(n, s)=\int_{0}^{1} \bar{T}(y, s) \sin (n \pi y) \mathrm{d} y, n=1,2, \ldots$.

Equation (31) can be written in the following equivalent form:

$$
\begin{aligned}
\widetilde{\bar{T}}_{s}(n, p)= & \frac{F_{1}(s)}{n \pi}+\frac{(-1)^{n+1} F_{2}(s)}{n \pi}-\frac{\left[F_{1}(s)+(-1)^{n+1} F_{2}(s)\right]}{n \pi} \\
& \times\left[1-\frac{(n \pi)^{2}}{\operatorname{Pr}} \frac{1}{\left(s^{\beta}+\left((n \pi)^{2} / \operatorname{Pr}\right)\right)+Q \operatorname{Pr}^{-1} s^{\beta-1}}\right] .
\end{aligned}
$$

Taking inverse Laplace transform of equation (32), we obtain

$$
\begin{aligned}
\widetilde{T}_{s}(n, t)= & \frac{f_{1}(t)}{n \pi}+\frac{(-1)^{n+1} f_{2}(t)}{n \pi}-\frac{\left[f_{1}(t)+(-1)^{n+1} f_{2}(t)\right]}{n \pi} \\
& *\left[\delta(t)-(n \pi)^{2} \sum_{m=0}^{\infty} \frac{(-Q)^{m}}{\operatorname{Pr}^{m+1}} G_{\beta,(\beta-1) m, m+1}\left(-\frac{(n \pi)^{2}}{\operatorname{Pr}}, t\right)\right],
\end{aligned}
$$

where "*” represents the convolution product, $\delta(t)$ is the direct delta function, and $G_{a, b, c}(\cdot)$ is the generalized G-function of Lorenzo and Hartley [49] defined as

$$
G_{a, b, c}(d, t)=L^{-1}\left\{\frac{p^{b}}{\left(p^{a}-d\right)^{c}}\right\}, \quad R(p)>0, R(a c-b)>0,\left|\frac{d}{p^{a}}\right|<1 .
$$

Taking the inverse sine-Fourier transform of equation (33), we obtain the temperature field

$$
\begin{aligned}
T(y, t)= & f_{1}(t)(1-y)+f_{2}(t) y-2 \sum_{n=1}^{\infty} \frac{1}{n \pi}\left[f_{1}(t)+(-1)^{n+1} f_{2}(t)\right] \\
& *\left[\delta(t)-(n \pi)^{2} \sum_{m=0}^{\infty} \frac{(-Q)^{m}}{\operatorname{Pr}^{m+1}} G_{\beta,(\beta-1) m, m+1}\left(-\frac{(n \pi)^{2}}{\operatorname{Pr}}, t\right)\right] \sin (n \pi y) .
\end{aligned}
$$

For the ordinary case when $\beta=1$, we have 


$$
\begin{aligned}
T(y, t)= & f_{1}(t)(1-y)+f_{2}(t) y-2 \sum_{n=1}^{\infty} \frac{1}{n \pi}\left[f_{1}(t)+(-1)^{n+1} f_{2}(t)\right] \\
& *\left[\delta(t)-\frac{(n \pi)^{2}}{\operatorname{Pr}} \exp \left(-\frac{(n \pi)^{2}+Q}{\operatorname{Pr}} t\right)\right] \sin (n \pi y) .
\end{aligned}
$$

For the special case, when $Q=0$, the similar solution is recovered as obtained in equation (35) in [12].

3.2. Velocity Field. Applying the Laplace transform to equations (27) and (15), using initial condition (13), we obtain

$$
(1+\lambda s) s^{\alpha} \bar{u}(y, s)=\left(1+\lambda_{r} s\right) \frac{\partial^{2} \bar{u}(y, s)}{\partial y^{2}}+\operatorname{Gr}(1+\lambda s) s^{\alpha-1} \bar{T}(y, s),
$$

$$
\begin{aligned}
& \bar{u}(0, s)=G_{1}(s), \\
& \bar{u}(1, s)=G_{2}(s) .
\end{aligned}
$$

Applying the finite sine-Fourier transform to differential equation (37) subject to the conditions in equation (38), we obtain

$$
\begin{aligned}
& {\left[s^{\alpha}+\lambda s^{\alpha+1}+\left(1+\lambda_{r} s\right)(n \pi)^{2}\right] \widetilde{\bar{u}}_{s}(n, s)} \\
& \quad=(n \pi)\left(1+\lambda_{r} s\right)\left[G_{1}(s)+(-1)^{n+1} G_{2}(s)\right]+\operatorname{Gr}\left[s^{\alpha-1}+\lambda s^{\alpha}\right] \widetilde{\bar{T}}_{s}(n, s)
\end{aligned}
$$

Using equation (31) in equation (39), we write the expression in suitable form as

$$
\begin{aligned}
\widetilde{\bar{u}}_{s}(n, s)= & \frac{(n \pi)\left(1+\lambda_{r} s\right)\left[G_{1}(s)+(-1)^{n+1} G_{2}(s)\right]}{\left(s^{\alpha}+\lambda s^{\alpha+1}+\left(1+\lambda_{r} s\right)(n \pi)^{2}\right)} \\
& +\frac{\operatorname{Gr}(n \pi)}{\operatorname{Pr}} \frac{\left(s^{\alpha-1}+\lambda s^{\alpha}\right)\left[F_{1}(s)+(-1)^{n+1} F_{2}(s)\right]}{\left(s^{\alpha}+\lambda s^{\alpha+1}+\left(1+\lambda_{r} s\right)(n \pi)^{2}\right)} \frac{1}{\left(s^{\beta}+\left((n \pi)^{2} / \operatorname{Pr}\right)\right)+Q \operatorname{Pr}^{-1} s^{\beta-1}} .
\end{aligned}
$$

Equation (40) can be written in the following equivalent form by using the series formula $\left(1 /\left(z_{1}+b\right)\right)=\sum_{k=0}^{\infty}$ $\left((-1)^{k} z_{1}^{k} / b^{k+1}\right),\left|z_{1} / b\right|<1$ as

$$
\begin{aligned}
\tilde{\bar{u}}_{s}(n, s)= & \frac{G_{1}(s)}{n \pi}+\frac{(-1)^{n+1} G_{2}(s)}{n \pi}-\frac{\left[G_{1}(s)+(-1)^{n+1} G_{2}(s)\right]}{n \pi} \\
& \times \sum_{l=0}^{\infty} \frac{(-1)^{l}}{\lambda^{l+1}} \sum_{k=0}^{l} \frac{l !(n \pi)^{2(l-k)}}{k !(l-k) !}\left[\frac{s^{(\alpha+1) k-l-1}}{\left(s^{\alpha}+\left(\lambda_{r}(n \pi)^{2} / \lambda\right)\right)^{l+1}}+\frac{\lambda s^{(\alpha+1) k-l}}{\left(s^{\alpha}+\left(\lambda_{r}(n \pi)^{2} / \lambda\right)\right)^{l+1}}\right] \\
& +G r(n \pi)\left[F_{1}(s)+(-1)^{n+1} F_{2}(s)\right] \\
& \times \sum_{n=0}^{\infty} \frac{(-1)^{n}}{\lambda^{l+1}} \sum_{k=0}^{n} \frac{l !(n \pi)^{2(l-k)}}{k !(l-k) !}\left[\frac{s^{(\alpha+1) k-l-2}}{\left(s^{\alpha}+\left(\lambda_{r}(n \pi)^{2} / \lambda\right)\right)^{l+1}}+\frac{\lambda s^{(\alpha+1) k-l-1}}{\left(s^{\alpha}+\left(\lambda_{r}(n \pi)^{2} / \lambda\right)\right)^{l+1}}\right] \\
& \sum_{m=0}^{\infty} \frac{(-Q)^{m}}{\operatorname{Pr}^{m+1}} \frac{s^{(\beta-1) m}}{\left(s^{\beta}+\left((n \pi)^{2} / \operatorname{Pr}\right)\right)^{m+1} .}
\end{aligned}
$$


Taking the inverse Laplace transform of equation (41), we obtain

$$
\begin{aligned}
\widetilde{u}_{s}(n, t)= & \frac{g_{1}(t)}{n \pi}+\frac{(-1)^{n+1} g_{2}(t)}{n \pi}-\frac{1}{n \pi}\left[g_{1}(t)+(-1)^{n+1} g_{2}(t)\right] \\
& * \sum_{l=0}^{\infty} \frac{(-1)^{l}}{\lambda^{l+1}} \sum_{k=0}^{l} \frac{l !(n \pi)^{2(l-k)}}{k !(l-k) !}\left[G_{\alpha,(\alpha+1) k-l-1, l+1}\left(-\frac{\lambda_{r}(n \pi)^{2}}{\lambda}, t\right)+\lambda G_{\alpha,(\alpha+1) k-l, l+1}\left(-\frac{\lambda_{r}(n \pi)^{2}}{\lambda}, t\right)\right] \\
& +G r(n \pi)\left[f_{1}(t)+(-1)^{n+1} f_{2}(t)\right] \\
& * \sum_{l=0}^{\infty} \frac{(-1)^{l}}{\lambda^{l+1}} \sum_{k=0}^{l} \frac{l !(n \pi)^{2(l-k)}}{k !(l-k) !}\left[G_{\alpha,(\alpha+1) k-l-2, n+1}\left(-\frac{\lambda_{r}(n \pi)^{2}}{\lambda}, t\right)+\lambda G_{\alpha,(\alpha+1) k-l-1, m+1}\left(-\frac{\lambda_{r}(n \pi)^{2}}{\lambda}, t\right)\right] \\
& * \sum_{m=0}^{\infty} \frac{(-Q)^{m}}{\operatorname{Pr}^{m+1}} G_{\beta,(\beta-1) m, m+1}\left(-\frac{(n \pi)^{2}}{\operatorname{Pr}}, t\right) .
\end{aligned}
$$

Taking the inverse sine-Fourier transform of equation (42), we have

$$
\begin{aligned}
u(y, t)= & g_{1}(t)(1-y)+g_{2}(t) y-2 \sum_{n=1}^{\infty} \frac{1}{n \pi}\left[g_{1}(t)+(-1)^{n+1} g_{2}(t)\right] * \sum_{l=0}^{\infty} \frac{(-1)^{l}}{\lambda^{l+1}} \sum_{k=0}^{l} \frac{l !(n \pi)^{2(l-k)}}{k !(l-k) !} \\
& \cdot\left[G_{\alpha,(\alpha+1) k-l-1, l+1}\left(-\frac{\lambda_{r}(n \pi)^{2}}{\lambda}, t\right)+\lambda G_{\alpha,(\alpha+1) k-l, l+1}\left(-\frac{\lambda_{r}(n \pi)^{2}}{\lambda}, t\right)\right] \sin (n \pi y) \\
& +2 \mathrm{Gr} \sum_{n=1}^{\infty}\left[f_{1}(t)+(-1)^{n+1} f_{2}(t)\right] * \sum_{l=0}^{\infty} \frac{(-1)^{l}}{\lambda^{l+1}} \sum_{k=0}^{n} \frac{l !(n \pi)^{2(l-k)+1}}{k !(l-k) !} \times \\
& \cdot\left[G_{\alpha,(\alpha+1) k-l-2, n+1}\left(-\frac{\lambda_{r}(n \pi)^{2}}{\lambda}, t\right)+\lambda G_{\alpha,(\alpha+1) k-l-1, l+1}\left(-\frac{\lambda_{r}(n \pi)^{2}}{\lambda}, t\right)\right] \\
& * \sum_{m=0}^{\infty} \frac{(-Q)^{m}}{\operatorname{Pr}^{m+1}} G_{\beta,(\beta-1) m, m+1}\left(-\frac{(n \pi)^{2}}{\operatorname{Pr}}, t\right) \sin (n \pi y) .
\end{aligned}
$$




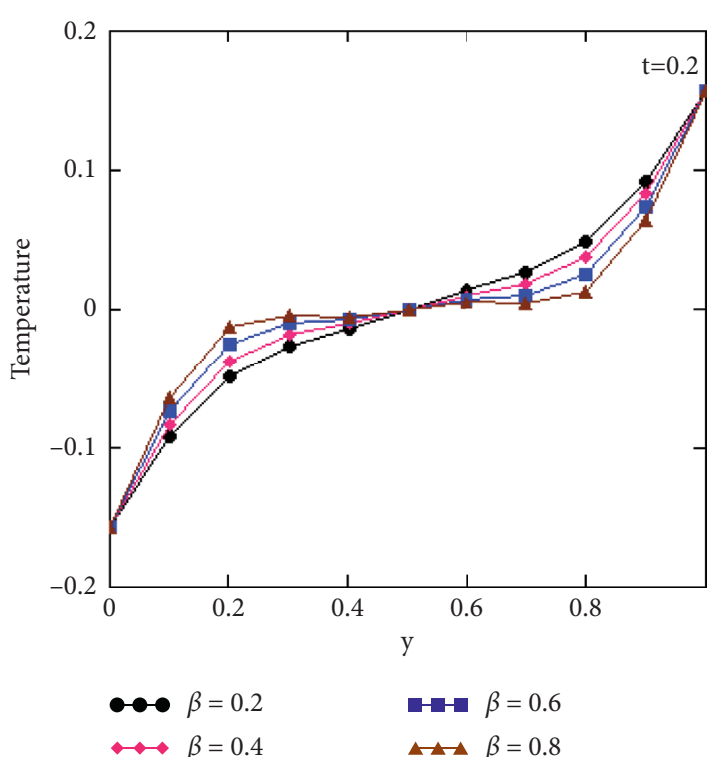

(a)

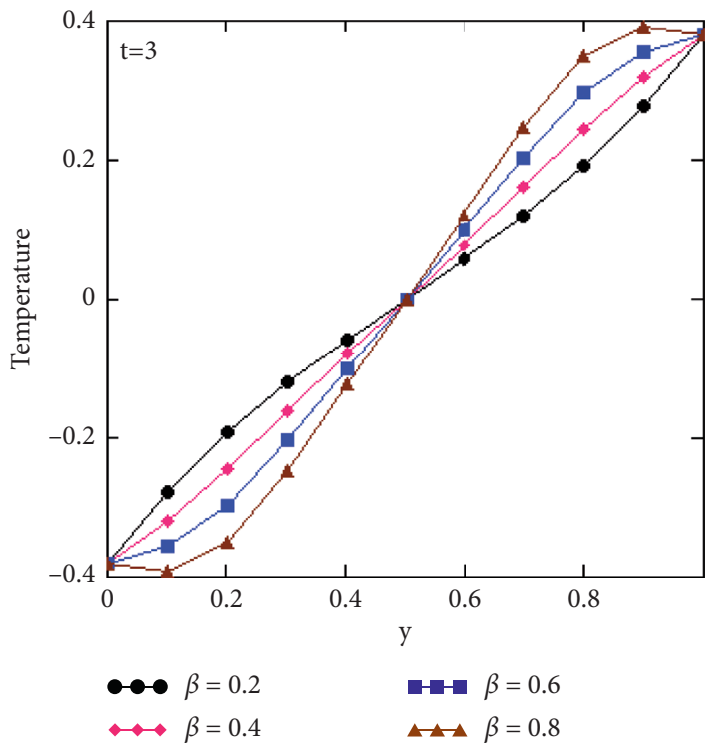

(c)

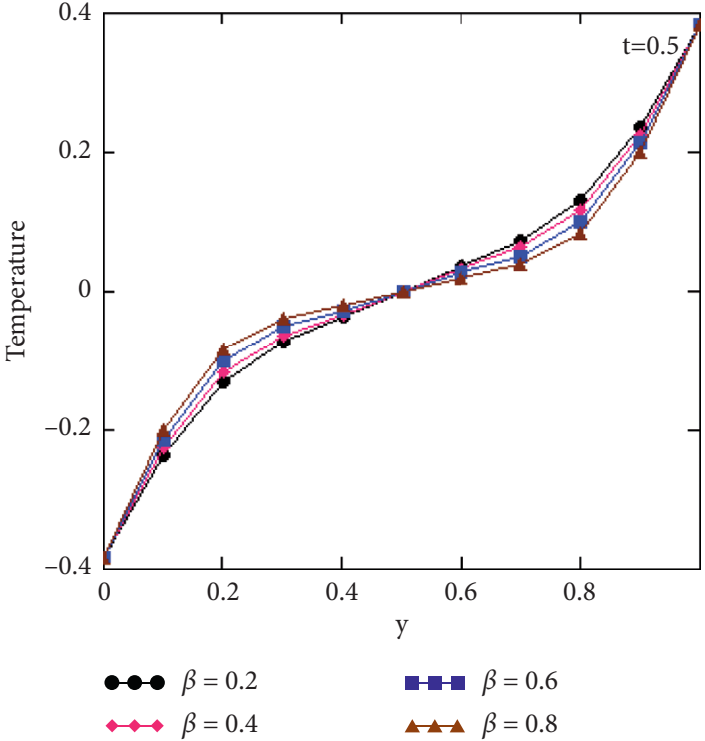

(b)

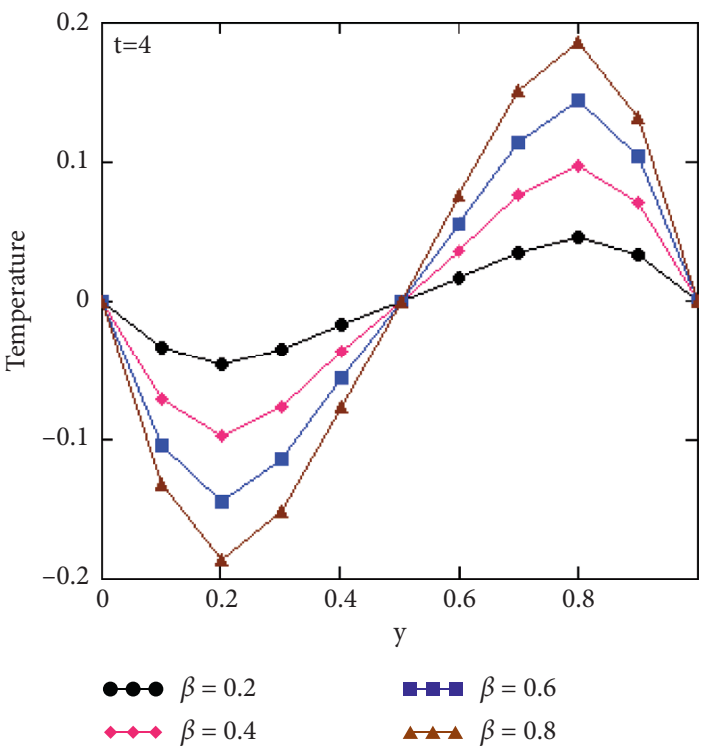

(d)

Figure 2: Profiles of dimensionless temperature versus $y$ for variation of $\beta$ at altered values of time $t, \operatorname{Pr}=20$ and $Q=0.5$, and $f_{1}(t)=-\sin (\pi t / 4)$ and $f_{2}(t)=\sin (\pi t / 4)$. 


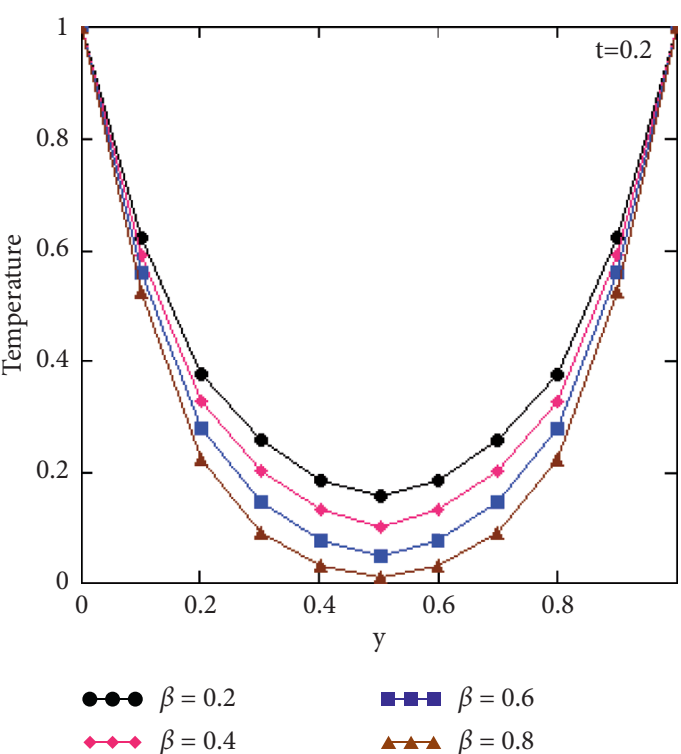

(a)

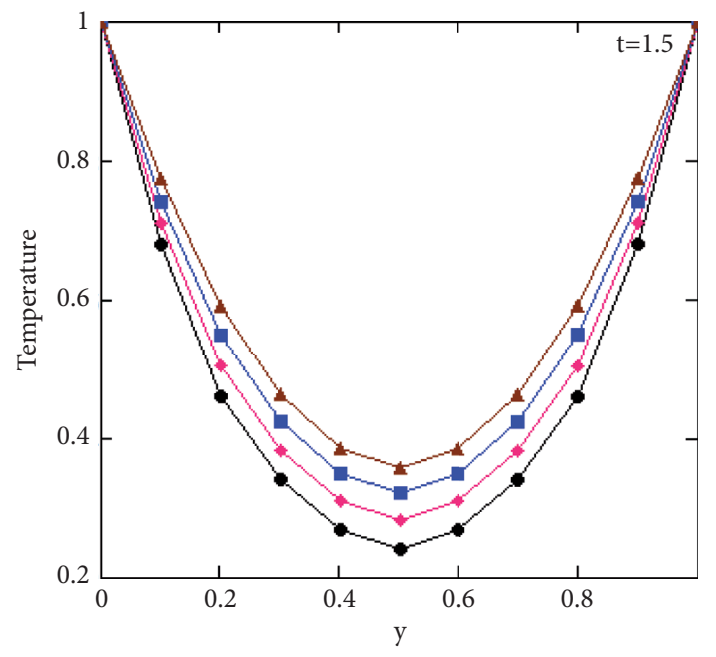

$\bullet \bullet \beta=0.2$

$\leftrightarrow \beta=0.4$

$$
\text { 믈 } \beta=0.6
$$$$
\leadsto \beta=0.8
$$

(c)

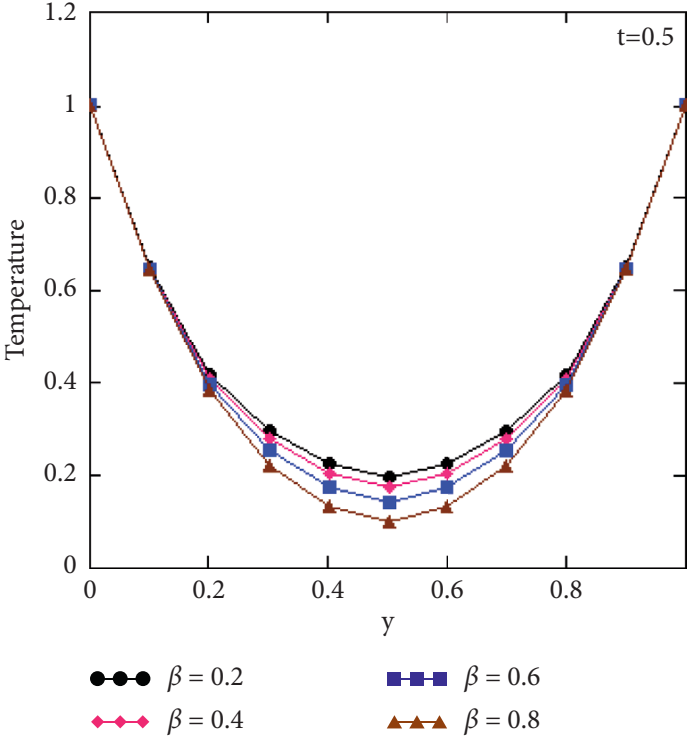

(b)

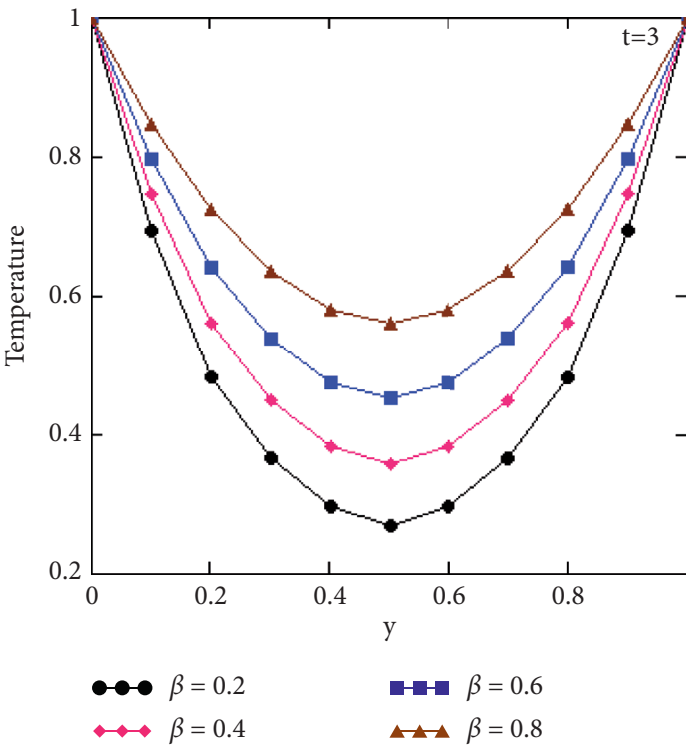

(d)

Figure 3: Profiles of dimensionless temperature versus $y$ for variation of $\beta$ at altered values of time $t, \operatorname{Pr}=20$ and $Q=0.5$, and $f_{1}(t)=H(t)=f_{2}(t)$. 


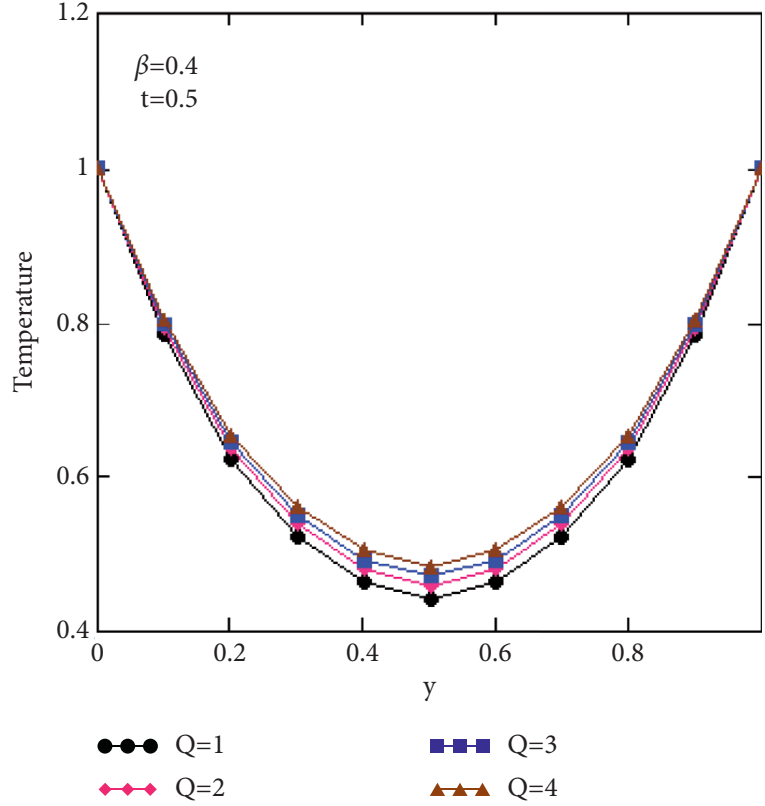

(a)

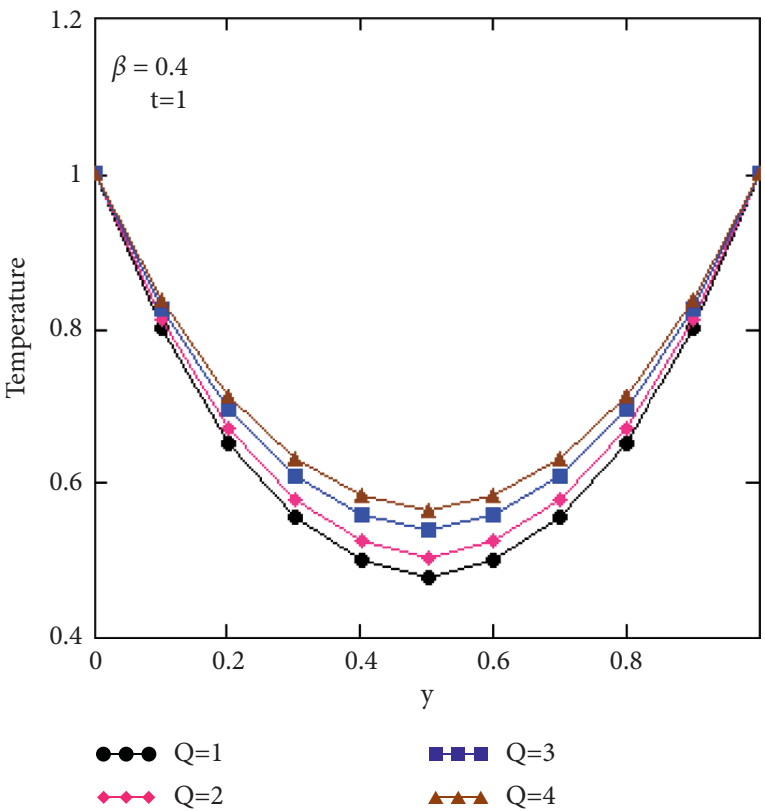

(b)

Figure 4: Profiles of dimensionless temperature versus $y$ for variation of $Q$ at altered values of time $t, \operatorname{Pr}=20$, and $f_{1}(t)=H(t)=f_{2}(t)$.

Remarks 1

(i) For the classical case corresponding to $\alpha=\beta=1$, we have the ordinary velocity field of the OBF from equation (43):

$$
\begin{aligned}
u(y, t)= & g_{1}(t)(1-y)+g_{2}(t) y-2 \sum_{n=1}^{\infty} \frac{1}{n \pi}\left[g_{1}(t)+(-1)^{n+1} g_{2}(t)\right] * \\
& \sum_{l=0}^{\infty} \frac{(-1)^{l}}{\lambda^{l+1}} \sum_{k=0}^{l} \frac{l !(n \pi)^{2(l-k)}}{k !(l-k) !}\left[G_{1,2 k-l-1, l+1}\left(-\frac{\lambda_{r}(n \pi)^{2}}{\lambda}, t\right)+\lambda G_{1,2 k-l, l+1}\left(-\frac{\lambda_{r}(n \pi)^{2}}{\lambda}, t\right)\right] \sin (n \pi y) \\
& +2 \frac{\operatorname{Gr}(n \pi)}{\operatorname{Pr}} \sum_{n=1}^{\infty}\left[f_{1}(q)+(-1)^{n+1} f_{2}(q)\right] \\
& * \sum_{l=0}^{\infty} \frac{(-1)^{l}}{\lambda^{l+1}} \sum_{k=0}^{n} \frac{l !(n \pi)^{2(l-k)}}{k !(l-k) !}\left[G_{1,2 k-l-2, n+1}\left(-\frac{\lambda_{r}(n \pi)^{2}}{\lambda}, t\right)+\lambda G_{1,2 k-l-1, l+1}\left(-\frac{\lambda_{r}(n \pi)^{2}}{\lambda}, t\right)\right] * \\
& \sum_{m=0}^{\infty} \frac{(-Q)^{m}}{\operatorname{Pr}^{m+1}} G_{1,0, m+1}\left(-\frac{(n \pi)^{2}}{\operatorname{Pr}}, t\right) \sin (n \pi y) .
\end{aligned}
$$

(ii) For fractional and classical Maxwell fluid when $\lambda_{r} \longrightarrow 0$ and $Q=0$ in equation (44), the similar solution is recovered as obtained in equations (43) and (44) in [12], respectively.

(iii) For fractional and classical viscous fluid when $\lambda \longrightarrow 0, \lambda_{r} \longrightarrow 0$, and $Q=0$ in equation (44), $w$ the similar solution is recovered as obtained in equation (45) and (46) in [12], respectively.

\section{Numerical Results and Discussion}

In order to examine the communicative changes on the thermal transport and flow profiles arising due to change of physical parameters, numerical estimations of the fluid temperature and velocity are figured and shown graphically in Figures 2-14.

In Figure 2, we exposed the effect of fractional parameter $\beta$ at different values of time $t$ and considering the functions $f_{1}(t)=-\sin (\pi t / 4)$ and $f_{2}(t)=\sin (\pi t / 4)$ at left and right plates, respectively. It is pointed out that, for small values of time $t$, the temperature is decreasing by increasing the values of fractional parameter $\beta$; after critical point, the influence becomes reversed for the large value of time. In Figure 3, we presented the effect of fractional parameter $\beta$ at different values of time $t$ and considering constant temperature at both plates. It has the similar influence like Figure 2. Also, we observed from this figure that the value of temperature at the 


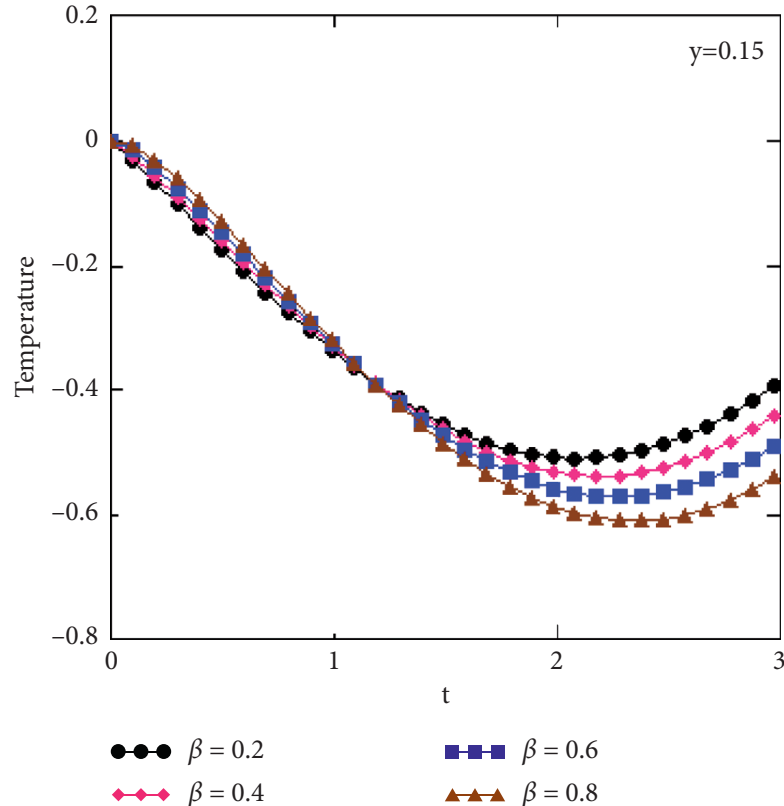

(a)

Figure 5: Profiles of dimensionless temperature verses $f_{1}(t)=-\sin (\pi t / 4)$ and $f_{2}(t)=\sin (\pi t / 4)$.

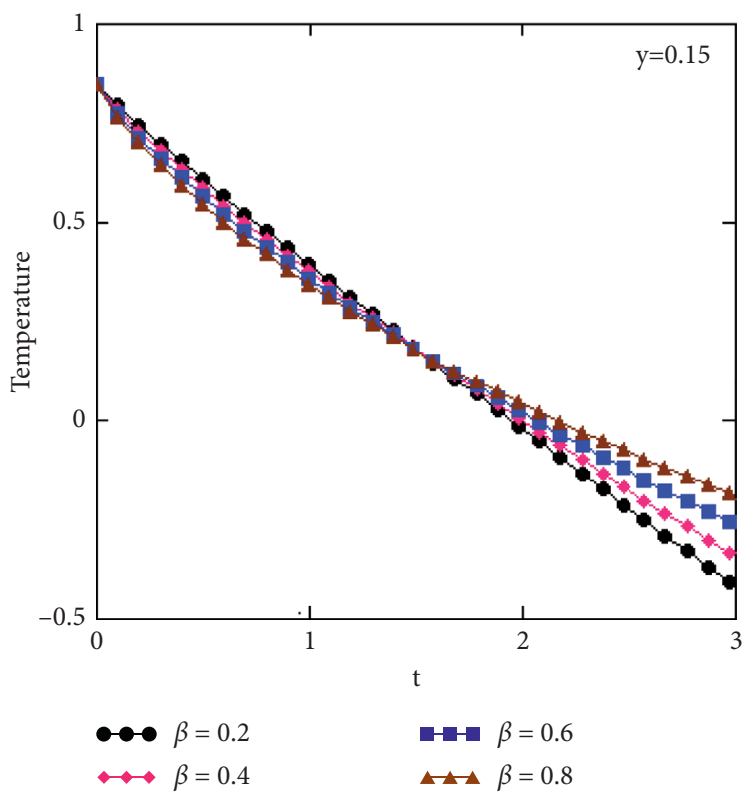

(a)

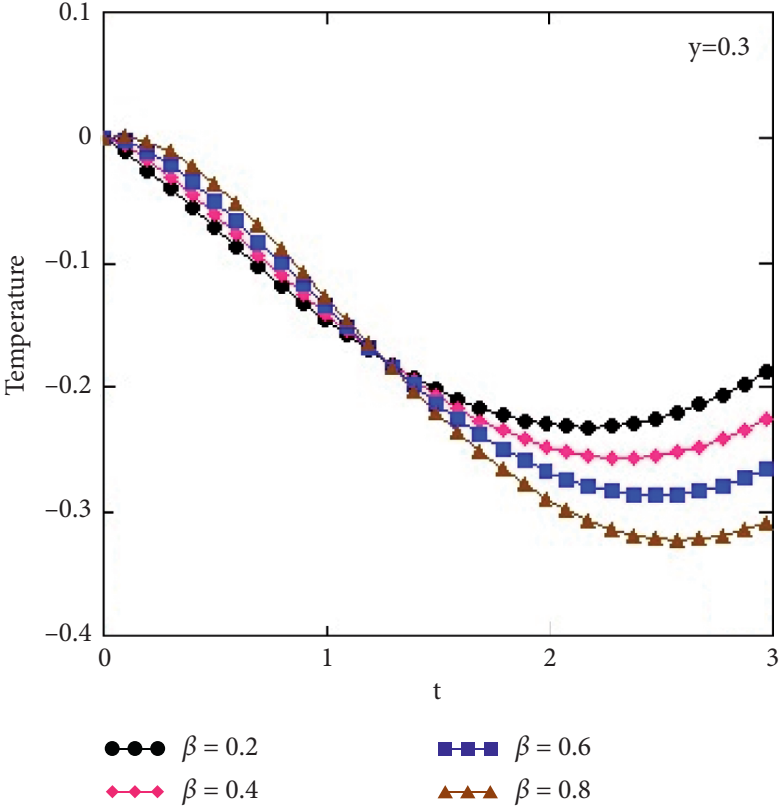

(b)

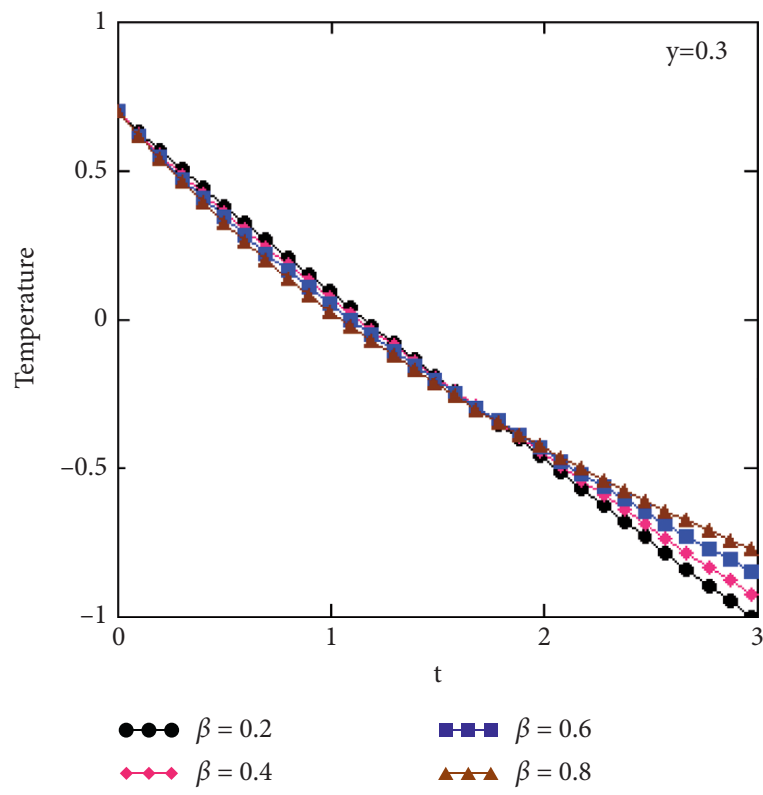

(b)

Figure 6: Profiles of dimensionless temperature verses $t$ for $\beta$ at two values of $y$ for $\operatorname{Pr}=20, Q=0.5$, and $f_{1}(t)=H(t)=f_{2}(t)$.

middle of the channel is low. The impact of absorption coefficient $Q$ on temperature profile is presented in Figure 4. We observed that temperature is an increasing function of $Q$ for small and large values of time $t$.

Figures 5 and 6 are presented to show the critical point for Figures 2 and 3, respectively.

In Figure 7 , we presented the effect of fractional parameter $\alpha$ at different values of time $t$, and $f_{1}(t)=H(t)$, $f_{2}(t)=e^{-5 t}$, and $g_{1}(t)=H(t)=g_{2}(t)$. From Figures $7(\mathrm{a})$ and $7(b)$, we observed that velocity profile increasing for small time $t$ corresponds to the large values of $\alpha$, while velocity profile increasing for large values of time $t$ corresponds to small values of $\alpha$. The effect of fractional parameter $\beta$ at different values of time $t, f_{1}(t)=f_{1}(t)=H(t)=$ $g_{1}(t)=g_{2}(t)$ and $f_{1}(t)=-H(t)$, and $f_{2}(t)=H(t)=$ $g_{1}(t)=g_{2}(t)$ is presented in Figures 8 and 9 , respectively. 


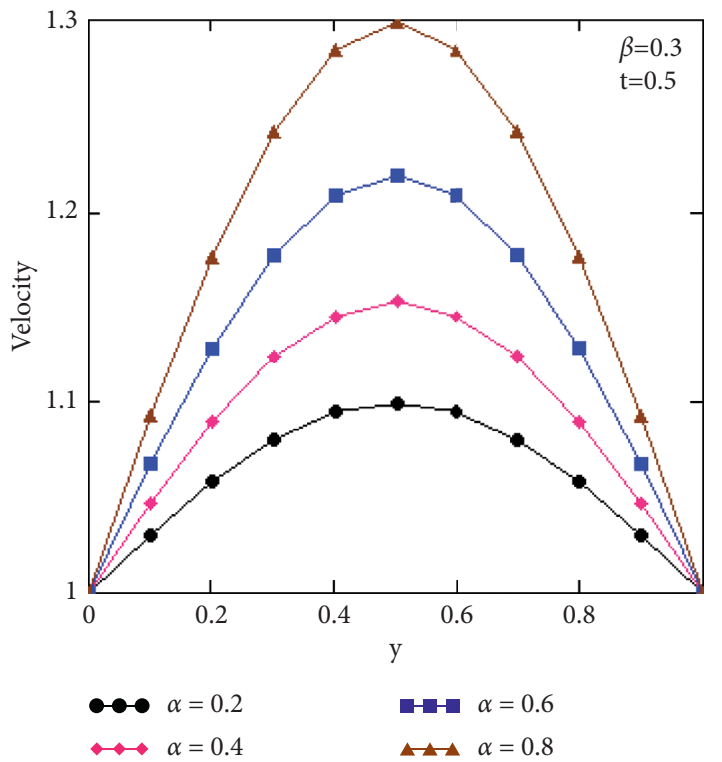

(a)

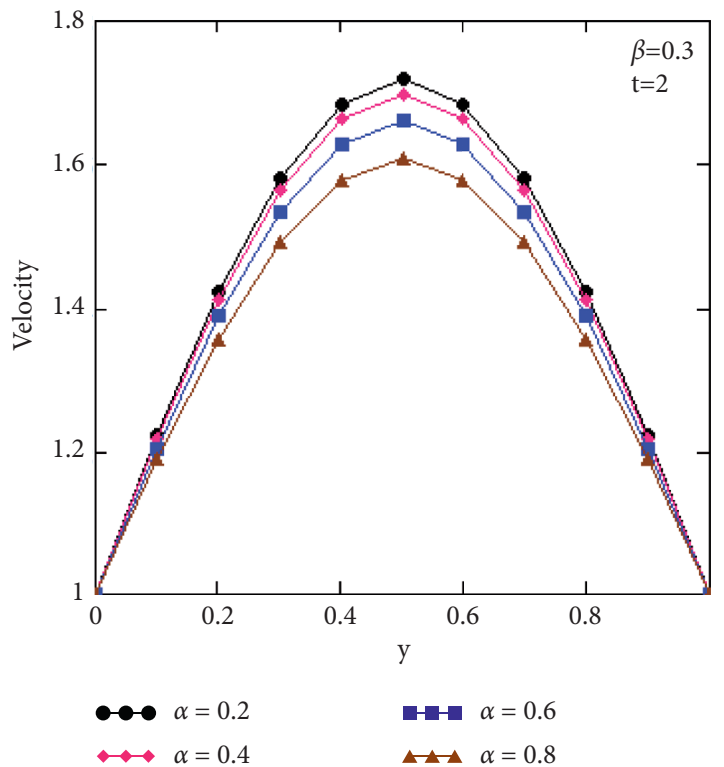

(c)

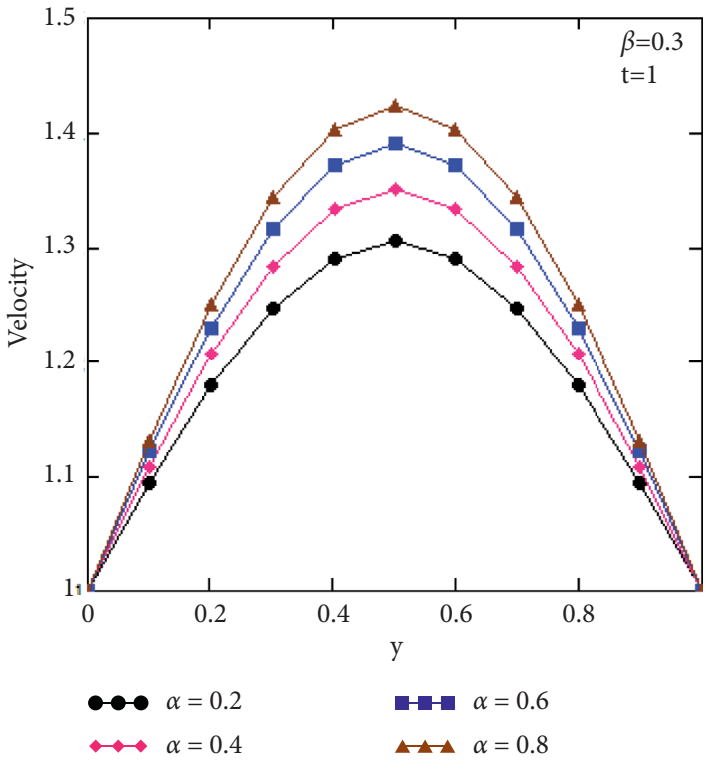

(b)

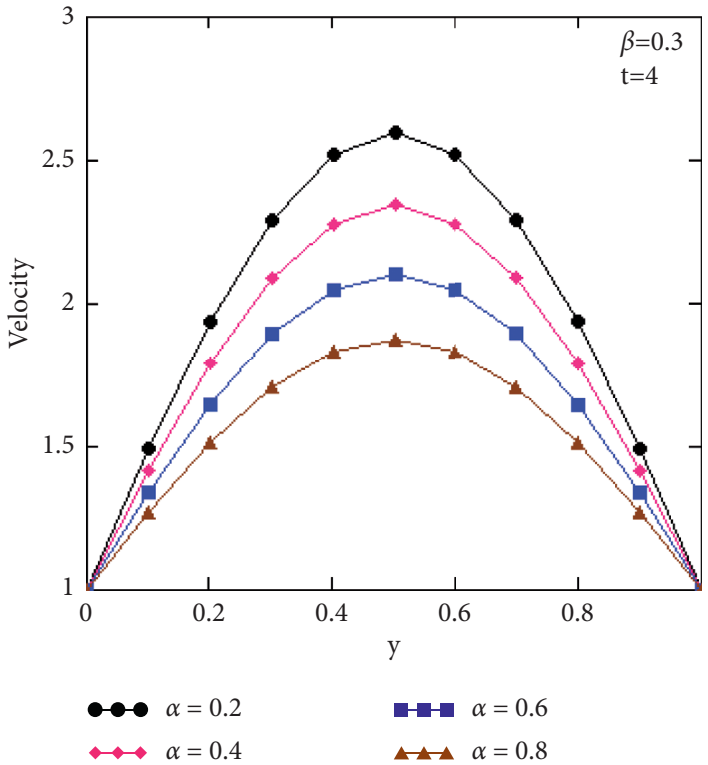

(d)

Figure 7: Profiles of dimensionless velocity versus $y$ for variation of $\alpha$ at altered values of time $t, \mathrm{Gr}=5, \lambda=2, \lambda_{r}=3, \operatorname{Pr}=20$, and $Q=0.5$, and $f_{1}(t)=H(t), f_{2}(t)=e^{-5 t}$, and $g_{1}(t)=H(t)=g_{2}(t)$.

From Figures 7-9, it is observed that, for small values of time velocity decreases by increasing the values of fractional parameters $\alpha$, while the influence is opposite for large values of time.

In order to find the critical value of time at which the influence becomes reversed, it is presented in Figure 10. The effects of relaxation and retardation parameters $\lambda$ and $\lambda_{r}$ and absorption coefficient $Q$ on velocity profile are presented in Figures 11-13. We observed that the velocity is an increasing function of $\lambda$ for small and large values of time $t$ because the relaxation time is the time needed by the fluid particles to adjust the flow motion of the fluid, while velocity is a decreasing function of $\lambda_{r}$ and $Q$ for small and large values of time $t$. The comparison between fractional Oldroyd-B, ordinary Oldroyd-B, fractional Maxwell, ordinary Maxwell, fractional viscous, and ordinary viscous fluids is presented in Figure 14. It is investigated that, for small time, ordinary fluids have greater velocity as compared to fractional fluids while for large time vice versa. Figures 15 and 16 are plotted to see the validity of our obtained results for temperature and velocity profiles by comparing to the $\mathrm{Na}$ et al. [12] outcomes. It can be seen from these figures that, by ignoring 


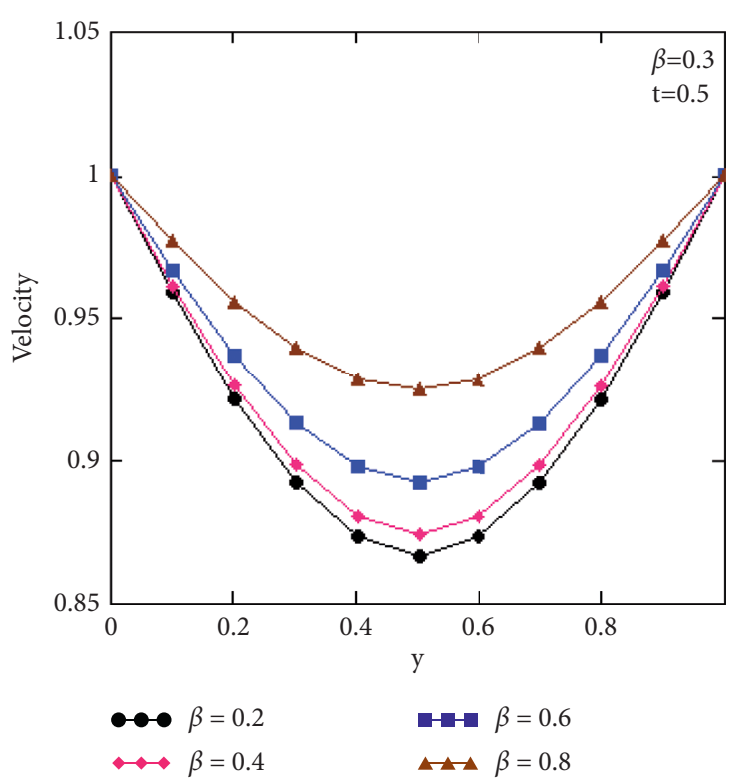

(a)

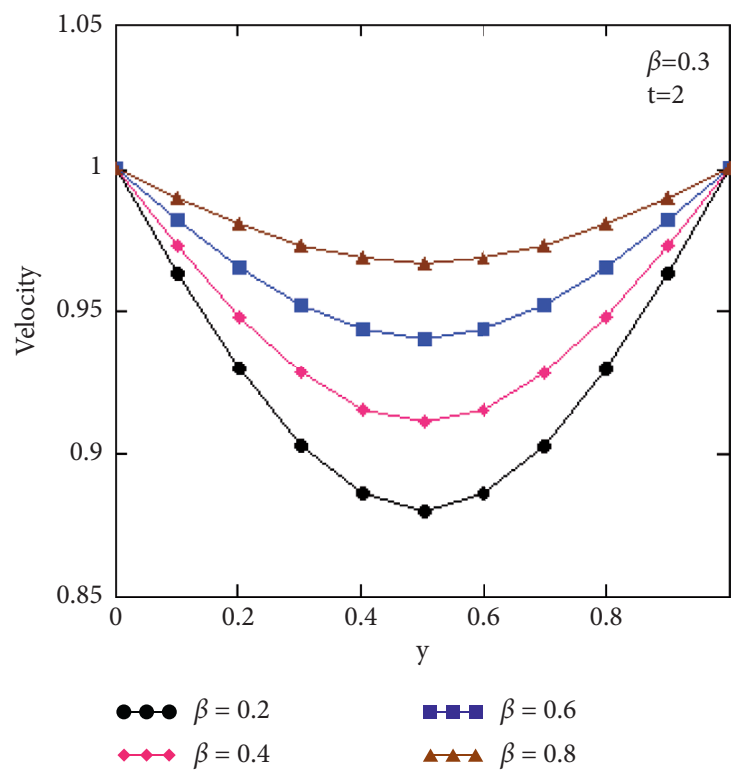

(c)

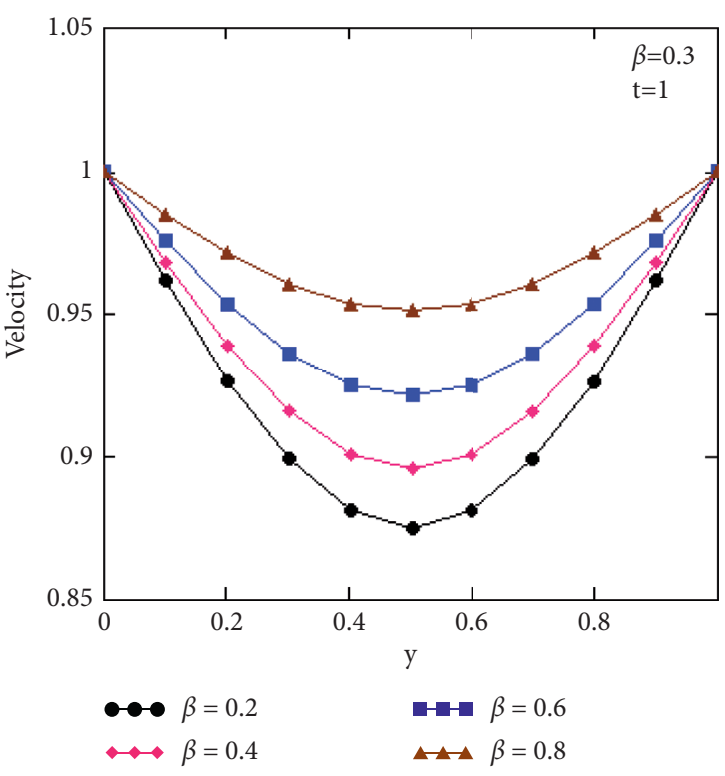

(b)

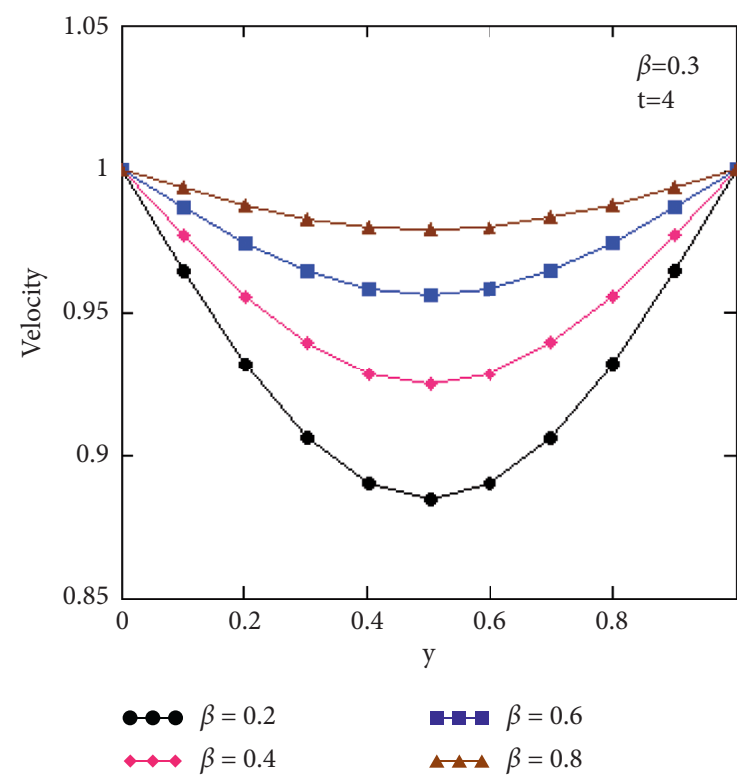

(d)

Figure 8: Profiles of dimensionless velocity versus $y$ for variation of $\alpha$ at altered values of time $t, \mathrm{Gr}=5, \lambda=2, \lambda_{r}=3, \operatorname{Pr}=20$, and $Q=0.5$, and $f_{1}(t)=-H(t)$ and $f_{2}(t)=H(t)=g_{1}(t)=g_{2}(t)$. 


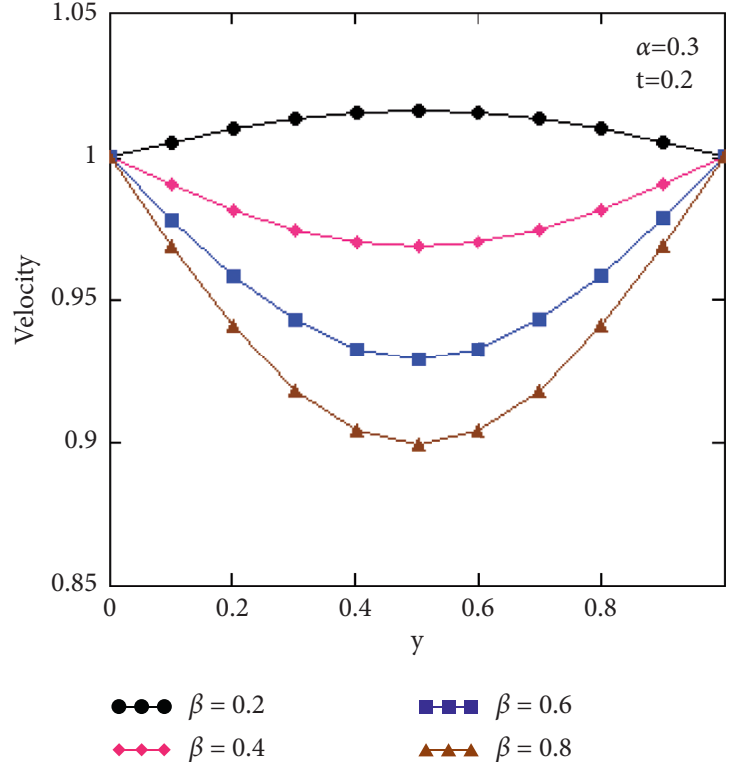

(a)

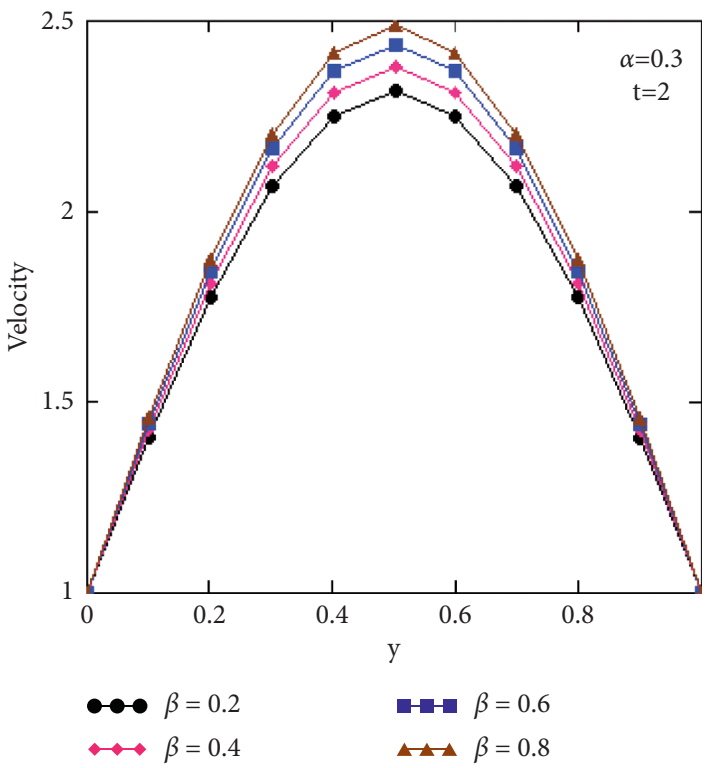

(c)

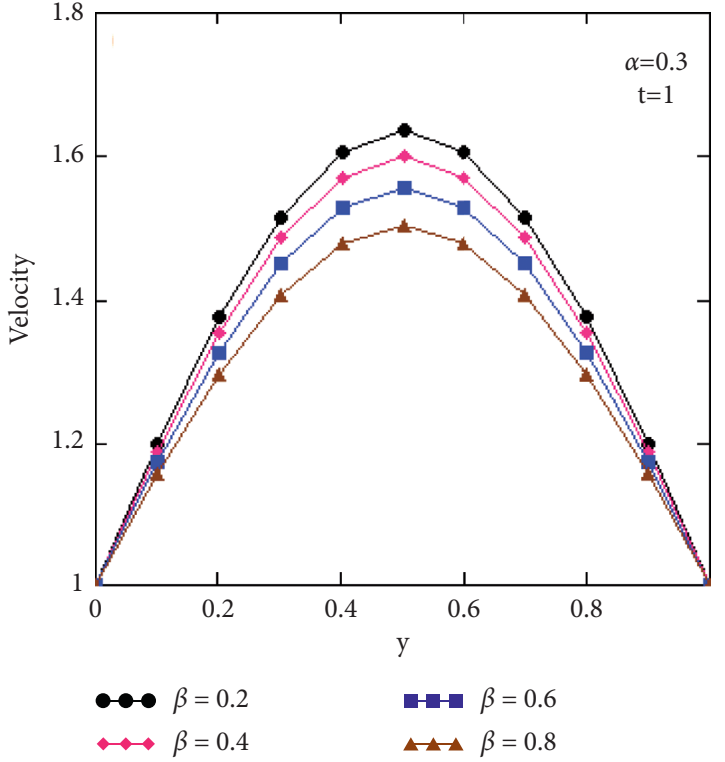

(b)

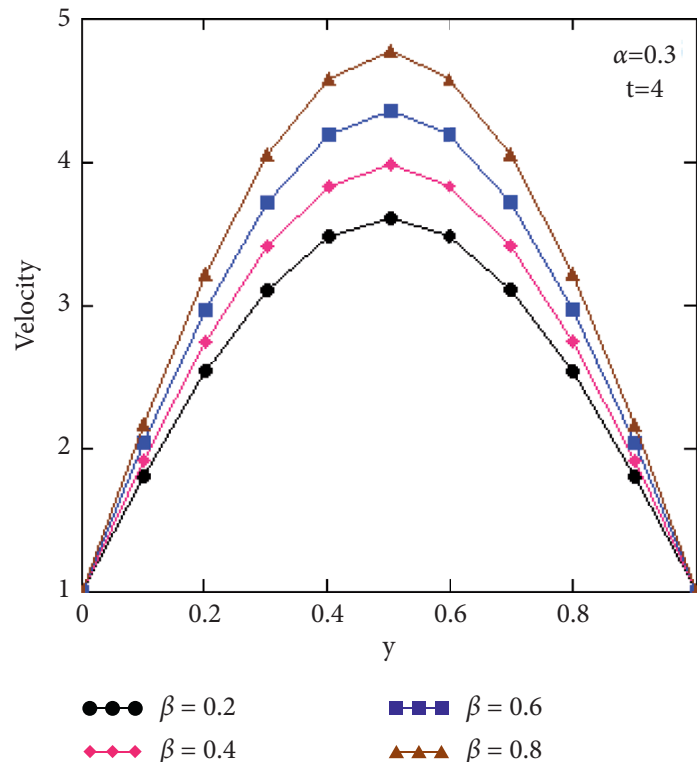

(d)

FiguRE 9: Profiles of dimensionless velocity versus $y$ for variation of $\beta$ at altered values of time $t, \mathrm{Gr}=5, \lambda=2, \lambda_{r}=3, \operatorname{Pr}=20, Q=0.5$, and $f_{1}(t)=f_{1}(t)=H(t)=g_{1}(t)=g_{2}(t)$. 


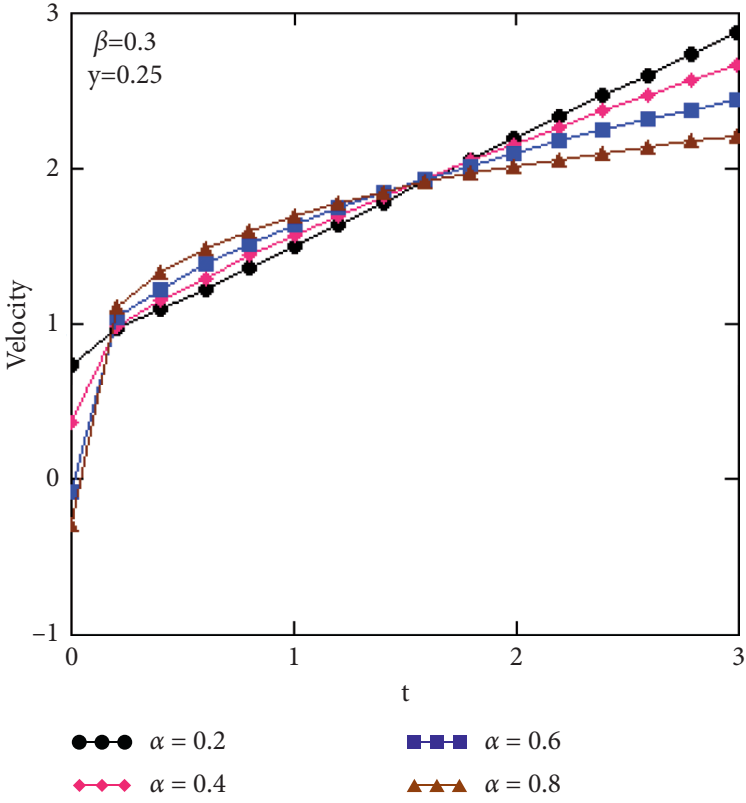

(a)

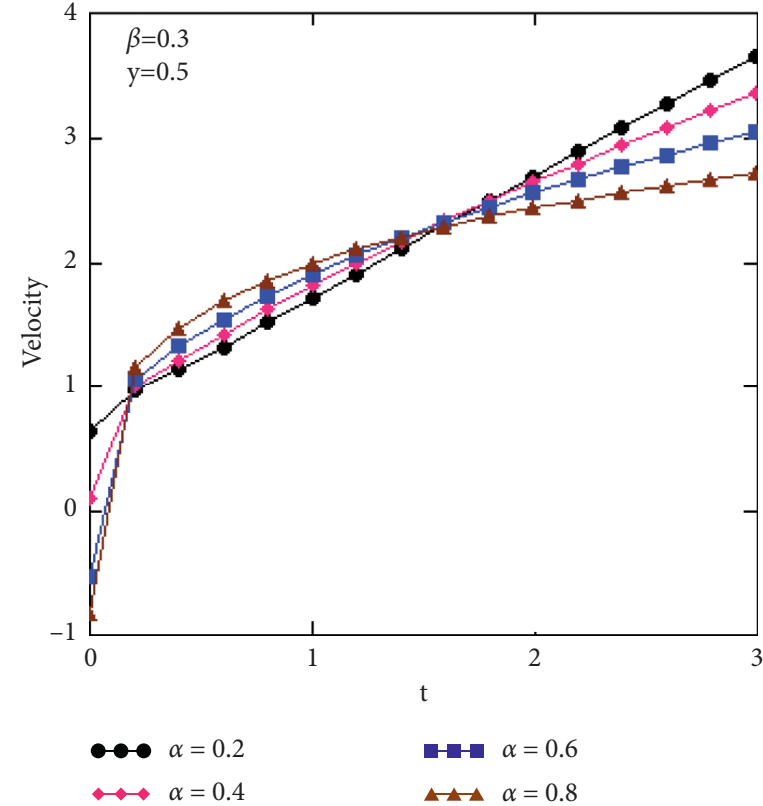

(b)

Figure 10: Profiles of dimensionless velocity versus $y$ for variation of $\alpha$ at altered values of time $t, \operatorname{Gr}=5, \lambda=2, \lambda_{r}=3, \operatorname{Pr}=20, Q=0.5$, and $f_{1}(t)=f_{1}(t)=H(t)=g_{1}(t)=g_{2}(t)$.

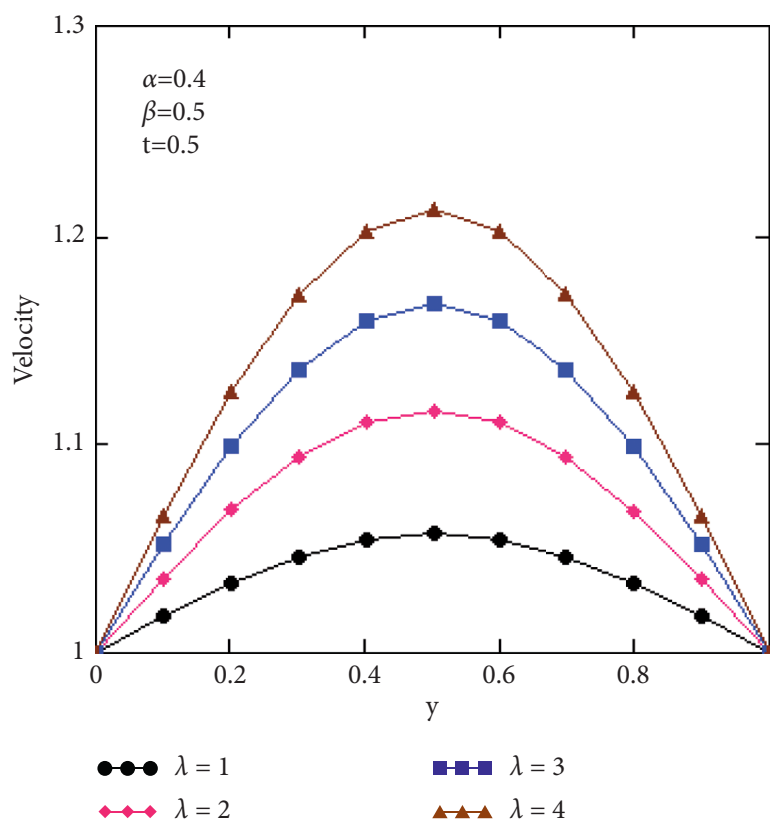

(a)

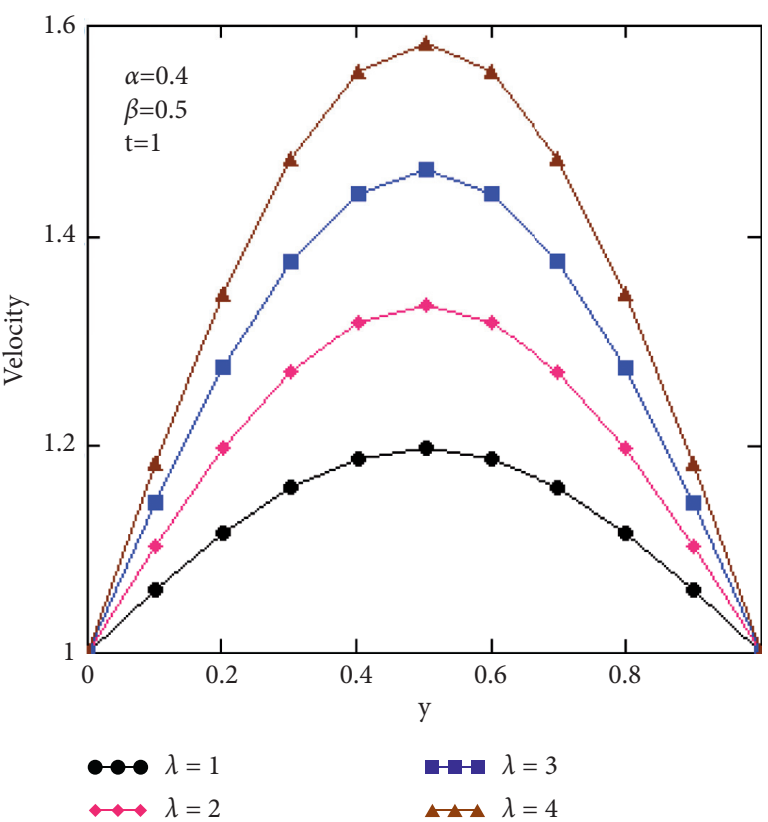

(b)

FIgURe 11: Profiles of dimensionless velocity versus $y$ for variation of $\lambda$ at altered values of time $t, \mathrm{Gr}=5, \lambda_{r}=3, \operatorname{Pr}=20, Q=0.5$, and $f_{1}(t)=f_{1}(t)=H(t)=g_{1}(t)=g_{2}(t)$. 


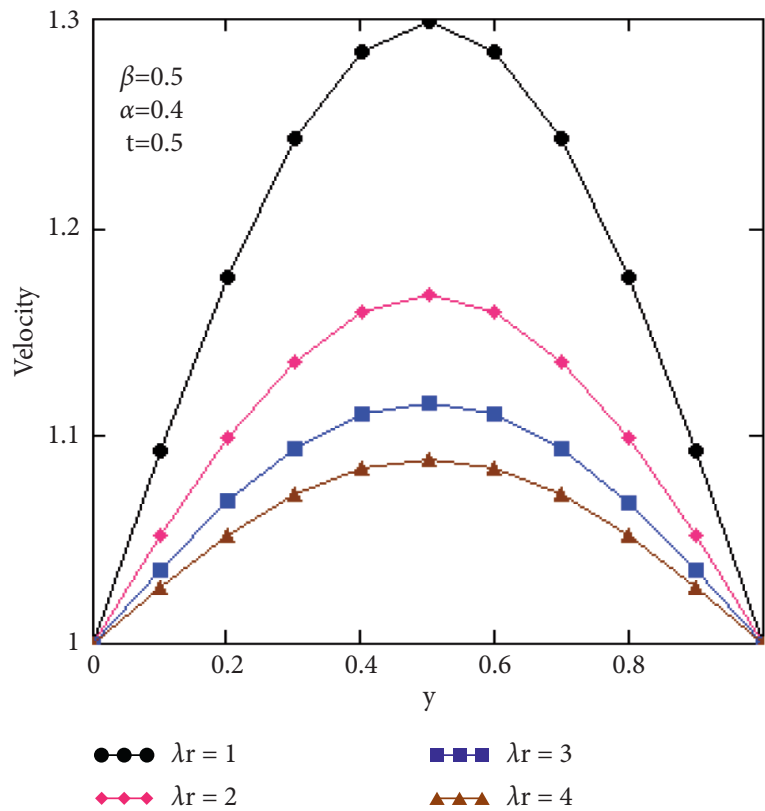

(a)

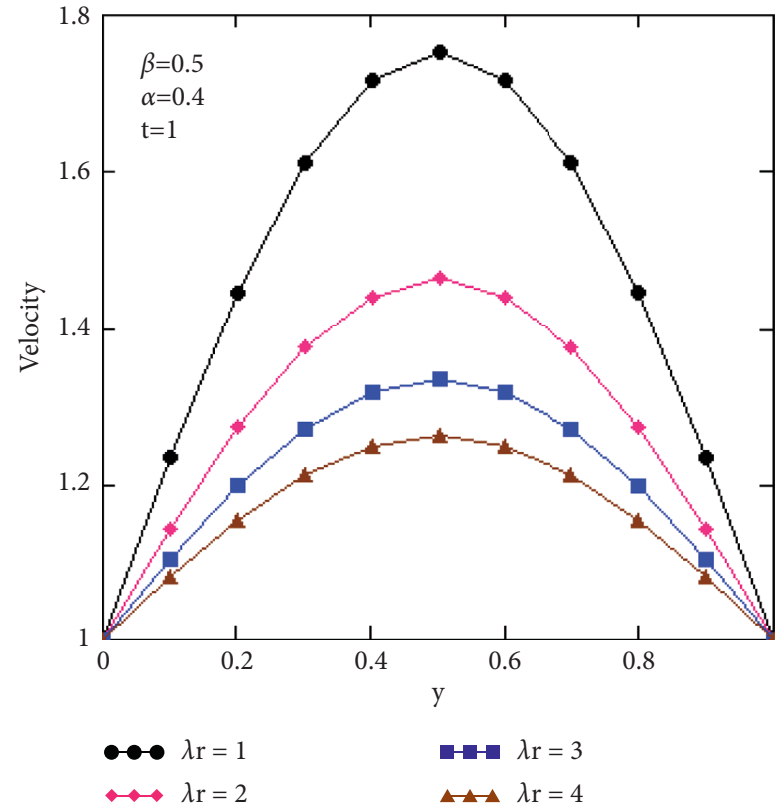

(b)

Figure 12: Profiles of dimensionless velocity versus $y$ for variation of $\lambda$ at altered values of time $t, \operatorname{Gr}=5, \lambda=2, \operatorname{Pr}=20, Q=0.5$, and $f_{1}(t)=f_{1}(t)=H(t)=g_{1}(t)=g_{2}(t)$.

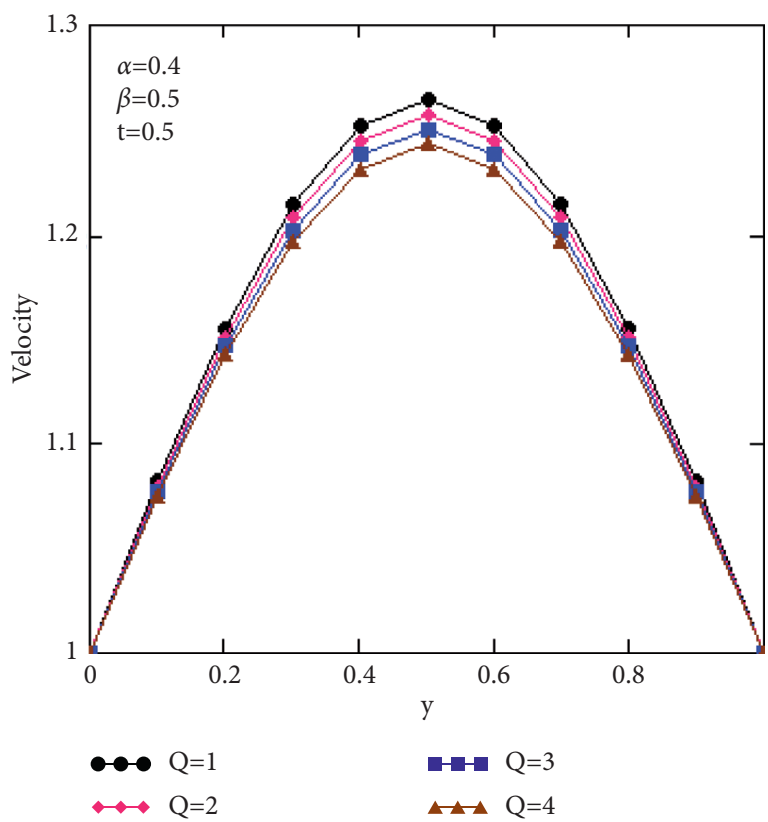

(a)

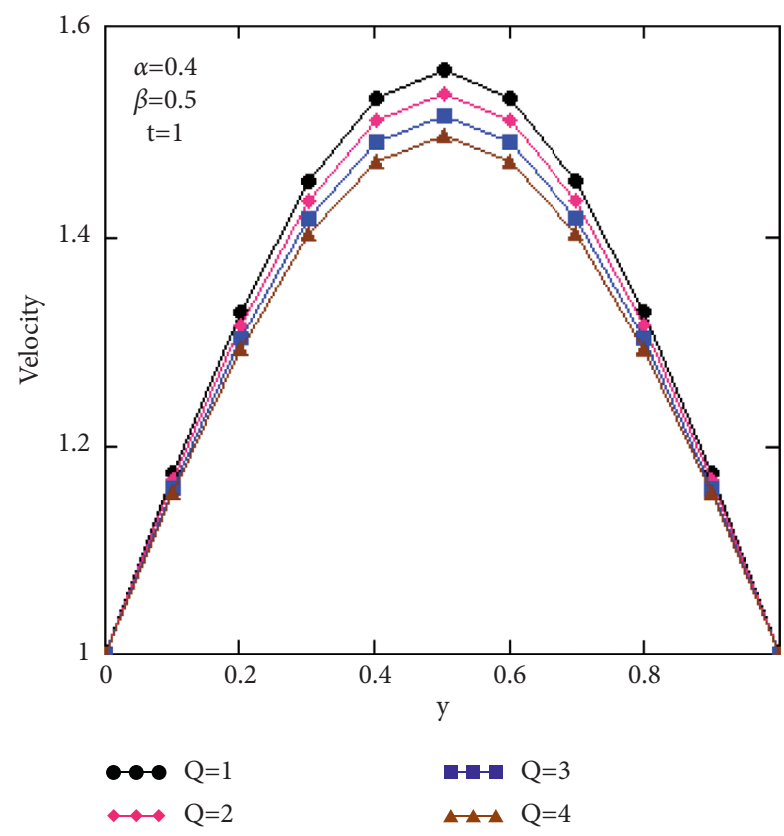

(b)

Figure 13: Profiles of dimensionless velocity versus $y$ for variation of $Q$ at altered values of time $t, \mathrm{Gr}=5, \lambda=2, \lambda_{r}=3, \operatorname{Pr}=20$, and $f_{1}(t)=f_{1}(t)=H(t)=g_{1}(t)=g_{2}(t)$. 


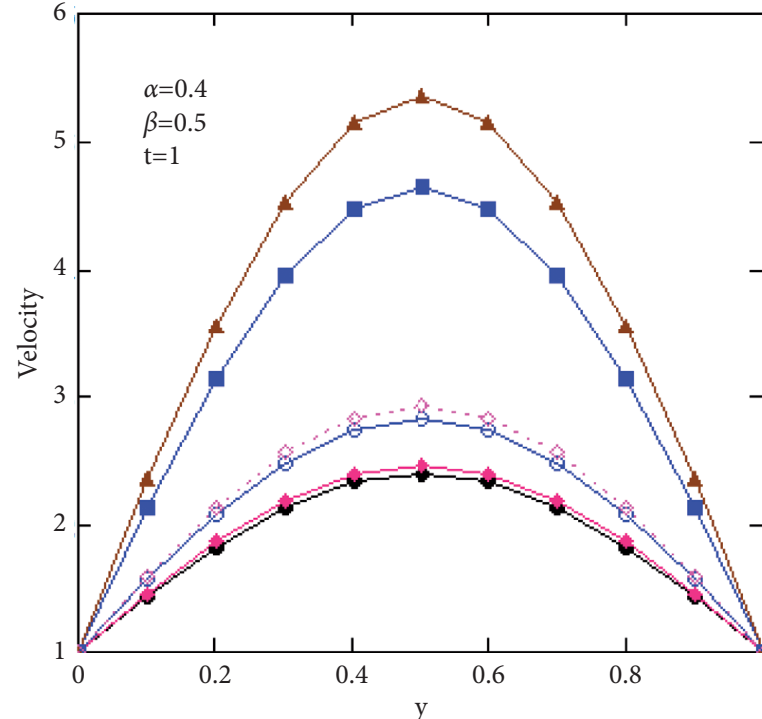

-๑ Fractional Oldroyd-B $\leftrightarrow$ Fractional Maxwell • Fractional Viscous

-1- Ordinary Oldroyd-B $\leftrightarrow \wedge$ Ordinary Maxwell $\diamond \diamond \diamond$ Ordinary Viscous

(a)

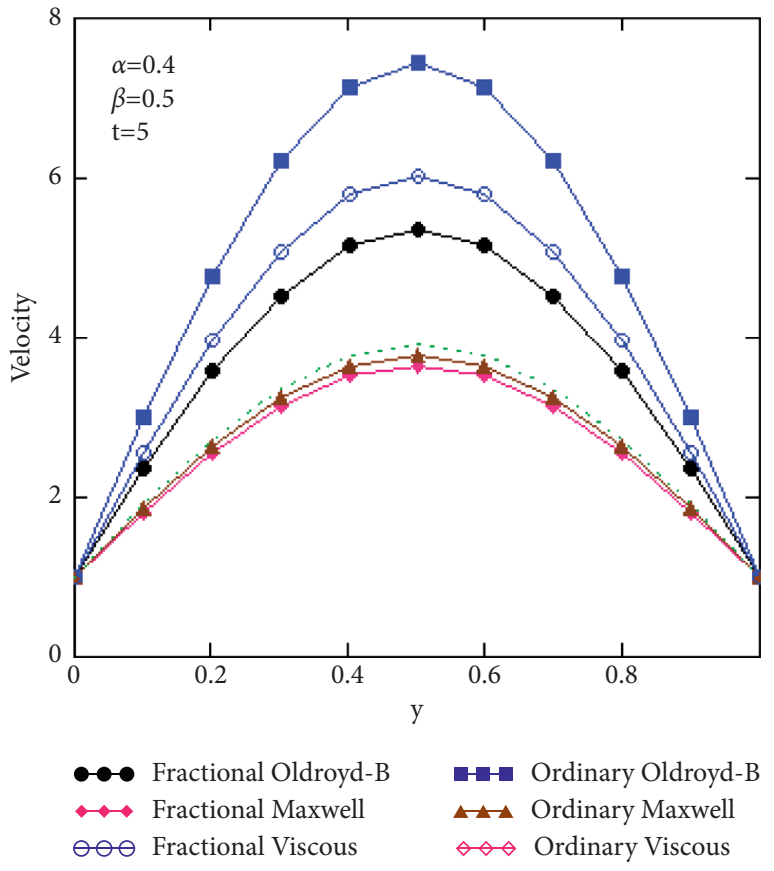

(b)

Figure 14: Comparisons between fractional Oldroy-B, ordinary Oldroyd-B, fractional Maxwell, ordinary Maxwell, fractional viscous, and ordinary viscous fluids at $\mathrm{Gr}=5, \operatorname{Pr}=20, Q=0.5$, and $f_{1}(t)=f_{1}(t)=H(t)=g_{1}(t)=g_{2}(t)$.

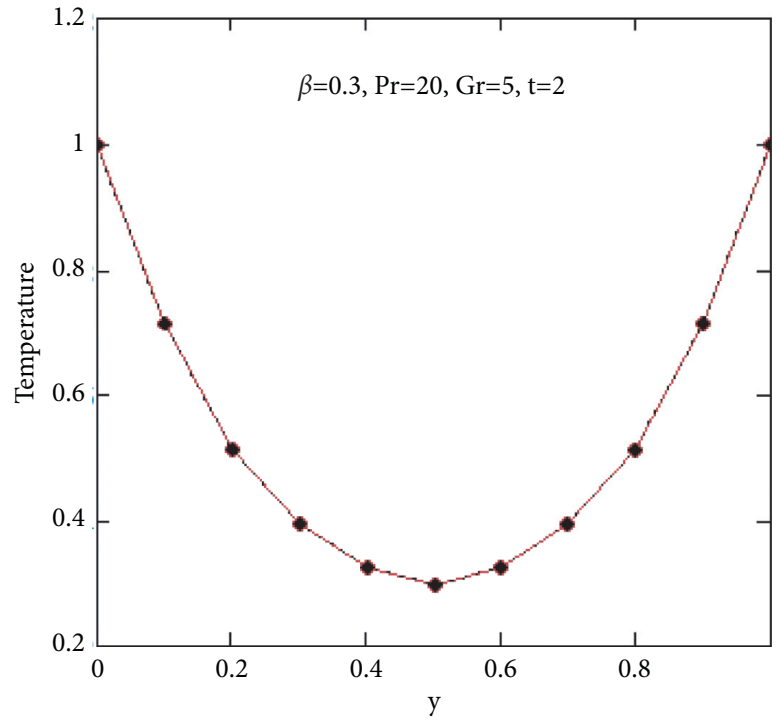

Results [12, Eq. (35)]

$\leftrightarrow$ Our results when $\mathrm{Q}=0$

FiguRE 15: Temperature profiles of our models compared with the temperature profile of [12] $f_{1}(t)=f_{1}(t)=H(t)=g_{1}(t)=g_{2}(t)$. 


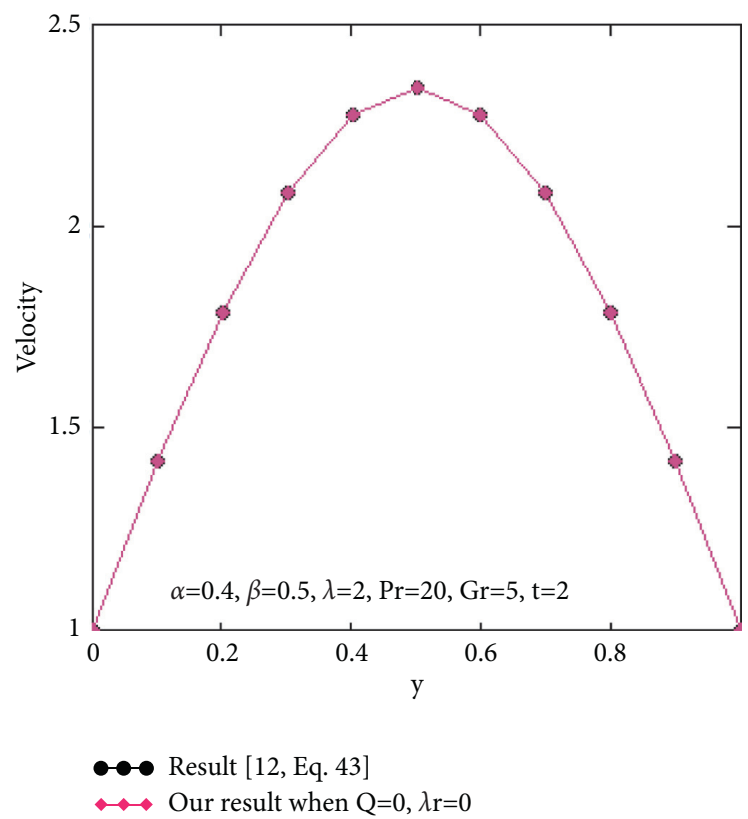

FIGURE 16: Velocity profiles of our models compared with the velocity profile of [12] $f_{1}(t)=f_{1}(t)=H(t)=g_{1}(t)=g_{2}(t)$.

the effects of $Q$ and $\lambda_{r}$, our results are identical to those obtained by [12].

\section{Conclusions}

The aim of this work is to study the free-convection flow of an OBF between two vertical parallel plates in the presence of heat generation or absorption subject to generalized boundary conditions. The Caputo time-fractional derivative is introduced by means of the generalized constitutive shear stress and damped thermal flux by using generalized Fourier's law. Closed form solutions of fractional differential equations are found by employing the Laplace and sineFourier transforms which are suitable for the boundary conditions. The obtained solutions are expressed in the form of generalized G-functions of Lorenzo and Hartley. The effects of fractional and physical parameters are graphically presented in Figures 2-14. Some key findings of our work are

(i) For small values of time, temperature is decreasing by increasing the values of fractional parameter and vice versa

(ii) Velocity decreases by increasing the values of fractional parameters for small values of time and vice versa

(iii) Velocity is an increasing and decreasing functions of $\lambda$ and $\lambda_{r}$ for small and large values of time $t$, respectively

(iv) Velocity is a decreasing functions of absorption coefficient $Q$ for small and large values of time $t$

(v) Velocity of Maxwell ordinary fluid is higher as compared to ordinary viscous and ordinary OBF for small times, while for large times velocity of fractional Maxwell fluid is higher as compared to fractional viscous and fractional OBF

(vi) Our obtained results for temperature and velocity profiles are identical to those obtained by [12]

\section{Nomenclature}

$u_{1}^{\mp}: \operatorname{Velocity}(\mathrm{m} / \mathrm{s})$

$T^{+}$: Temperature $(\mathrm{K})$

$t^{\mp}:$ Time $(\mathrm{sec})$

$g:$ Gravitational acceleration $\left(\mathrm{m} / \mathrm{s}^{2}\right)$

$k$ : Thermal conductivity $(W / \mathrm{mK})$

$c_{p}:$ Specific heat $(J / \mathrm{kgK})$

$Q_{0}$ : Heat absorption coefficient $\left(W / \mathrm{m}^{3} \mathrm{~K}\right)$

Gr: Thermal Grash of number

Pr: Prandtl number

$\lambda^{\mp}$ : Relaxation time (sec)

$\lambda_{r}^{\mp}:$ Retardation time $(\mathrm{sec})$

$\tau^{+}$: Shear stress $\left(\mathrm{kg} / \mathrm{ms}^{2}\right)$

$\mu$ : Dynamic viscosity $(\mathrm{kg} / \mathrm{ms})$

$\rho: \quad$ Density $\left(\mathrm{kg} / \mathrm{m}^{3}\right)$

$v$ : Kinematic viscosity $\left(\mathrm{m}^{2} / \mathrm{s}\right)$

$\beta_{T}$ : Thermal expansion coefficient $\left(\mathrm{K}^{-1}\right)$.

\section{Data Availability}

No data were used to support this study.

\section{Conflicts of Interest}

The authors declare that there are no conflicts of interest regarding the publication of this paper. 


\section{References}

[1] J. G. Oldroyd, "On the formulation of rheological equations of state," Proceedings of the Royal Society of London - Series A: Mathematical and Physical Sciences, vol. 200, no. 1063, pp. 523-541, 1950.

[2] K. R. Rajagopal and R. K. Bhatnagar, "Exact solutions for some simple flows of an Oldroyd-B fluid," Acta Mechanica, vol. 113, no. 1-4, pp. 233-239, 1995.

[3] T. Hayat, A. M. Siddiqui, and S. Asghar, "Some simple flows of an Oldroyd-B fluid," International Journal of Engineering Science, vol. 39, no. 2, pp. 135-147, 2001.

[4] C. Fetecau and C. Fetecau, "The first problem of Stokes for an Oldroyd-B fluid," International Journal of Non-linear Mechanics, vol. 38, no. 10, pp. 1539-1544, 2003.

[5] C. Fetecau and C. Fetecau, "Unsteady flows of Oldroyd-B fluids in a channel of rectangular cross-section," International Journal of Non-linear Mechanics, vol. 40, no. 9, pp. 1214-1219, 2005.

[6] C. Fetecau, S. C. Prasad, and K. R. Rajagopal, "A note on the flow induced by a constantly accelerating plate in an OldroydB fluid," Applied Mathematical Modelling, vol. 31, no. 4, pp. 647-654, 2007.

[7] H. Qi and M. Xu, "Stokes' first problem for a viscoelastic fluid with the generalized Oldroyd-B model," Acta Mechanica Sinica, vol. 23, no. 5, pp. 463-469, 2007.

[8] M. Khan, "The Rayleigh-Stokes problem for an edge in a viscoelastic fluid with a fractional derivative model," Nonlinear Analysis: Real World Applications, vol. 10, no. 5, pp. 3190-3195, 2009.

[9] S. Kakac, M. Aung, and R. Viskanta, Natural Convection: Fundamentals and Applications, Hemisphere Publishing Corporation, Washington, DC, USA, 1985.

[10] A. K. Singh, H. R. Gholami, and V. M. Soundalgekar, "Transient free convection flow between two vertical parallel plates," Heat and Mass Transfer, vol. 31, no. 5, pp. 329-331, 1996.

[11] M. Narahari, Transient Free Convection Flow between Two Long Vertical Parallel Plates with Constant Temperature and Mass Diffusion, Proceedings of the World Congress on Engineering, London, UK, 2008.

[12] W. Na, N. A. Shah, I. Tlili, and I. Siddique, "Maxwell fluid flow between vertical plates with damped shear and thermal flux: free convection," Chinese Journal of Physics, vol. 65, pp. 367-376, 2020.

[13] U. N. Zaib, N. A. Shah, I. Tlili, S. Ullah, and M. Nazar, "Natural convection flow of second grade fluid with thermal radiation and damped thermal flux between vertical channels," Alexandria Engineering Journal, vol. 58, no. 4, pp. 1119-1125, 2019.

[14] A. Hajizadeh, N. A. Shah, S. I. A. Shah, I. L. Animasaun, M. Rahimi-Gorji, and I. M. Alarifi, "Free convection flow of nanofluids between two vertical plates with damped thermal flux," Journal of Molecular Liquids, vol. 289, Article ID 110964, 2019.

[15] Z. Shao, N. A. Shah, I. Tlili, U. Afzal, and M. S. Khan, "Hydromagnetic free convection flow of viscous fluid between vertical parallel plates with damped thermal and mass fluxes," Alexandria Engineering Journal, vol. 58, no. 3, pp. 989-1000, 2019.

[16] G. S. Seth, N. Mahto, R. Tripathi, and A. Bhattacharyya, "Unsteady hydromagnetic flow formation with Hall effect due to time-dependent free stream in a rotating medium," Journal of Nature Science and Sustainable Technology, vol. 11, no. 3, pp. 197-211, 2018.

[17] G. S. Seth, A. Bhattacharyya, and R. Tripathi, "Effect of Hall current on MHD natural convection heat and mass transfer flow of rotating fluid past a vertical plate with ramped wall temperature," Frontiers in Heat and Mass Transfer, vol. 9, no. $21,2017$.

[18] N. Ahmed, D. Vieru, C. Fetecau, and N. A. Shah, "Convective flows of generalized time-nonlocal nanofluids through a vertical rectangular channel," Physics of Fluids, vol. 30, no. 5, Article ID 52002, 2018.

[19] B. K. Jha, K. A. Samaila, and A. O. Ajibade, "Natural convection flow of heat generating/absorbing fluid near a vertical plate with ramped temperature," Journal of Encapsulation and Adsorption Sciences, vol. 2, no. 4, pp. 61-68, 2012.

[20] B. K. Jha and B. Aina, "Impact of Heat generation/absorption on MHD mixed convection flow in a vertical tube having time periodic boundary condition: steady-periodic regime," Heat Pipe Science and Technology, An International Journal, vol. 7, no. 1-2, pp. 123-147, 2016.

[21] B. K. Jha, M. O. Oni, and B. Aina, "Steady fully developed mixed convection flow in a vertical micro-concentric-annulus with heat generating/absorbing fluid: an exact solution," Ain Shams Engineering Journal, vol. 9, no. 4, pp. 1289-1301, 2018.

[22] F. Saba, N. Ahmed, S. Hussain, U. Khan, S. Mohyud-Din, and M. Darus, "Thermal analysis of nanofluid flow over a curved stretching surface suspended by carbon nanotubes with internal heat generation," Applied Sciences, vol. 8, no. 3, p. 395, 2018.

[23] M. Aleem, M. I. ASjad, A. Shaheen, and I. Khan, "MHD Influence on different water based nanofluids ( $\mathrm{TiO} 2, \mathrm{Al} 2 \mathrm{O} 3$, $\mathrm{CuO})$ in porous medium with chemical reaction and Newtonian heating," Chaos, Solitons \& Fractals, vol. 130, Article ID 109437, 2020.

[24] N. A. Shah, A. H. Seikh, I. Tlili et al., "Natural convection of bio-nanofluid between two vertical parallel plates with damped shear and thermal flux," Journal of Molecular Liquids, vol. 296, no. 15, p. 111575, 2019.

[25] M. Ramzan, Z. Un Nisa, M. Ahmad, and M. Nazar, "Unsteady free convective magnetohydrohynamics flow of a casson fluid through a channel with double diffusion and ramp temperature and concentration," Mathematical Methods and Applied Sciences, vol. 2021, pp. 1-11, 2021.

[26] I. Siddique, N. A. Shah, and K. A. Abro, "Thermography of ferromagnetic Walter's-B fluid through varying thermal stratification," South African Journal of Chemical Engineering, vol. 36, pp. 118-126, 2021.

[27] I. Siddique, K. Sadiq, I. Khan, and K. S. Nisar, "Nanomaterials in convection flow of nanofluid in upright channel with gradients," Journal of Materials Research and Technology, vol. 11, pp. 1411-1423, 2021.

[28] X. Qiang, I. Siddique, K. Sadiq, and N. A. Shah, "Double diffusive MHD convective flows of a viscous fluid under influence of the inclined magnetic field, source/sink and chemical reaction," Alexandria Engineering Journal, vol. 6, no. 59, pp. 4171-4181, 2020.

[29] J. Hristov, "A transient flow of a non-Newtonian fluid modelled by a mixed time-space derivative: an improved integral-balance approach," in Mathematical Methods in Engineering-Theory, K. Tas, D. Baleanu, and J. A. T. Machado, Eds., Springer International Publishing, Berlin, Germany, 2018. 
[30] I. Podlubny, Fractional Differential Equations, Academic, San Diego, CA, USA, 1999.

[31] S. Hyder Ali Muttaqi Shah, "Some accelerated flows of generalized Oldroyd-B fluid between two side walls perpendicular to the plate," Nonlinear Analysis: Real World Applications, vol. 10, no. 4, pp. 2146-2150, 2009.

[32] A. Akgül and I. Siddique, "Analysis of MHD Couette flow by fractal-fractional differential operators," Chaos, Solitons \& Fractals, vol. 146, Article ID 110893, 2021.

[33] A. Akgül and I. Siddique, "Novel applications of the magnetohydrodynamics couple stress fluid flows between two plates with fractal-fractional derivatives," Numerical Methods for Partial Differential Equations, vol. 37, no. 3, pp. 2178-2189, 2021.

[34] C. Fetecau, C. Fetecau, M. Kamran, and D. Vieru, "Exact solutions for the flow of a generalized Oldroyd-B fluid induced by a constantly accelerating plate between two side walls perpendicular to the plate," Journal of Non-newtonian Fluid Mechanics, vol. 156, no. 3, pp. 189-201, 2009.

[35] I. Siddique and A. Akgül, "Analysis of blood liquor model via nonlocal and singular constant proportional Caputo hybrid differential operator," Mathematical Methods in the Applied Sciences, vol. 66, 2021.

[36] M. Khan, T. Hayat, and S. Asghar, "Exact solution for MHD flow of a generalized Oldroyd-B fluid with modified Darcy's law," International Journal of Engineering Science, vol. 44, no. 5-6, pp. 333-339, 2006.

[37] I. Siddique and Z. Sajid, "Exact solutions for the unsteady axial flow of non-Newtonian fluids through a circular cylinder," Communications in Nonlinear Science and Numerical Simulation, vol. 16, no. 1, pp. 226-238, 2011.

[38] Y. Liu, L. Zheng, and X. Zhang, "Unsteady MHD Couette flow of a generalized Oldroyd-B fluid with fractional derivative," Computers \& Mathematics with Applications, vol. 61, no. 2, pp. 443-450, 2011.

[39] D. Bose and U. Basu, "Unsteady incompressible flow of a generalized Oldroyd-B fluid between two oscillating infinite parallel plates in presence of a transverse magnetic field," Applied Mathematics, vol. 6, no. 1, pp. 106-115, 2015.

[40] J. Zhao, L. Zheng, X. Zhang, F. Liu, and X. Chen, "Unsteady natural convection heat transfer past a vertical flat plate embedded in a porous medium saturated with fractional Oldroyd-B fluid," Journal of Heat Transfer, vol. 1, Article ID 12501, 2017.

[41] A. A. Zafar, M. B. Riaz, N. A. Shah, and M. A. Imran, "Influence of non-integer-order derivatives on unsteady unidirectional motions of an Oldroyd-B fluid with generalized boundary conditions," The European Physical Journal Plus, vol. 133, no. 3, p. 127, 2018.

[42] J. Zhang, F. Liu, and V. V. Anh, "Analytical and numerical solutions of a two-dimensional multi-term time-fractional Oldroyd-B model," Numerical Methods for Partial Differential Equations, vol. 35, no. 3, pp. 875-893, 2019.

[43] M. B. Riaz and S. T. Saeed, "Comprehensive analysis of integer-order, Caputo-Fabrizio (CF) and Atangana-Baleanu (ABC) fractional time derivative for MHD Oldroyd-B fluid with slip effect and time dependent boundary condition," Discrete \& Continuous Dynamical Systems - S, vol. 14, no. 10, p. 3719, 2021.

[44] B. O. Wang, M. Javaid, M. Imran, M. Tahir, and C. Y. Jung, "Semi Analytical solutions for fractional Oldroyd-B fluid through rotating annulus," IEEE Access, vol. 7, 2019.

[45] M. Kamran, M. Imran, and M. Athar, "Exact solutions for the unsteady rotational flow of an Oldroyd-B fluid with fractional derivatives induced by a circular cylinder," Meccanica, vol. 48, no. 5, pp. 1215-1226, 2013.

[46] I. Siddique and S. M. Bukhari, "Analysis of the effect of generalized fractional Fourier's and Fick's laws on convective flows of non-Newtonian fluid subject to Newtonian heating," The European Physical Journal Plus, vol. 135, no. 1, p. 45, 2020.

[47] I. Siddique, I. Tlili, S. M. Bukhari, and Y. Mahsud, "Heat transfer analysis in convective flows of fractional second grade fluids with caputo-fabrizio and atangana-baleanu derivative subject to newtonion heating," Mechanics of Time-Dependent Materials, vol. 32, pp. 1-21, 2020.

[48] K. Sadiq, F. Jarad, I. Siddique, and B. Ali, "Soret and radiation effects on mixture of ethylene glycol-water (50\%-50\%) based Maxwell nanofluid flow in an upright channel," Complexity, vol. 2021, Article ID 5927070, 12 pages, 2021.

[49] C. F. Lorenzo and T. T. Hartley, "Generalized functions for fractional calculus," ResearchGate, Berlin, Germany, NASA/ TP-1999-209424, 1999.

[50] J. Zhao, L. Zheng, X. Zhang, and F. Liu, "Unsteady convection heat and mass transfer of a fractional oldroyd-b fluid with chemical reaction and heat source/sink effect," Heat Transfer Research, vol. 49, no. 13, pp. 1231-1246, 2018.

[51] M. B. Riaz, A. Atangana, and T. Abdeljawad, "Local and nonlocal differential operators: a comparative study of heat and mass transfers in mhd oldroyd-b fluid with ramped wall temperature," Fractals, vol. 28, no. 8, Article ID 2040033, 2020.

[52] J. E. Caffyn and G. W. S. Blair, "Significance of power-law relations in rheology," Nature, vol. 155, pp. 171-172, 1955.

[53] Y. Povstenko, Fractional Thermoelasticity, Solid Mechanics and its Applications, Springer International Publishing, Cham, Switzerland, 2015.

[54] J. Hristov, "Derivatives with non-singular kernels. from the CaputoFabrizio definition and beyond: appraising analysis with emphasis on diffusion models," in Frontiers in Fractional Calculus, S. Bhalekar, Ed., pp. 269-340, Bentham Science Publishers, Sharjah, UAE, 1st edition, 2017.

[55] M. Caputo, "Linear models of dissipation whose Q is almost frequency independent--II," Geophysical Journal International, vol. 13, no. 5, pp. 529-539, 1967.

[56] T. M. Atanackovic', S. Pilipovic', B. Stankovic', and D. Zorica, "Fractional calculus with applications in mechanics: vibrations and diffusion processes," in Applied Mathematics, N. Challamel, Ed., ISTE Ltd., John Wiley \& Sons, Inc., London, UK, 2014. 